\title{
Fifty WSRT highlights
}

\section{Richard Strom}

ASTRON

Oude Hoogeveensedijk 4, 7991 PD Dwingeloo, The Netherlands

E-mail: strom@astron.nl

\section{Thijs van der Hulst}

Kapteyn Astronomical Institute, University of Groningen, Landleven 12, 9747AD Groningen,

The Netherlands

E-mail: j.m.van.der.hulst@rug.nl

\section{Arnold van Ardenne}

ASTRON

Oude Hoogeveensedijk 4, 7991 PD Dwingeloo, The Netherlands

E-mail: ardenne@astron.nl 


\section{Chapter 18 Fifty WSRT highlights}

1972

1973

Richard Strom', Thijs van der Hulst², Arnold van Ardenne ${ }^{3}$

Ger de Bruyn felt that a leading telescope should produce about one outstanding result each year. $\mathrm{He}$ maintained a list of exciting science coming out of the WSRT, and when last updated it numbered 50 noteworthy results, about one per year with the 5 oth anniversary of the telescope soon upon us. Largely based on that with a few additions and modifications, the following pages represent WSRT milestones most of which are represented by an image as published by those who did the research. Most of the highlights concern astronomical results, though 10\% fall in the categories of hard-/software development. Each highlight is presented with a minimum of descriptive text: the pictures should speak for themselves. Enjoy the almost abstract shapes, shades and colors which rolled out of the WSRT (a search of the Astrophysics Data System (ADS) will help satisfy the curious) relish these WSRT highlights! 
Software (mapping, display), 1970

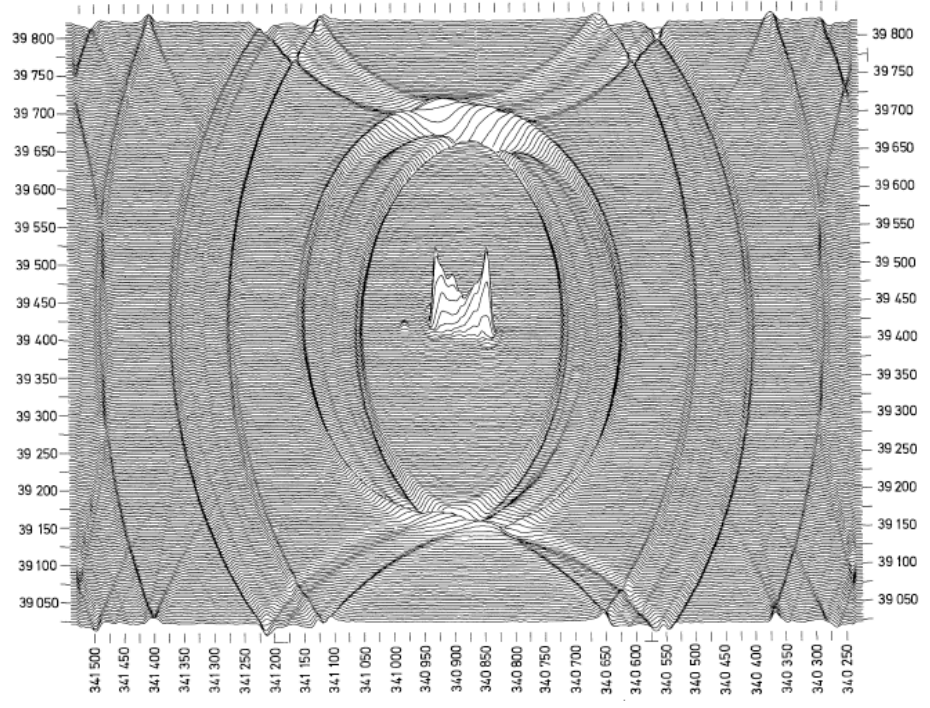

Cyg X-1 radio flares \& identification, 1971

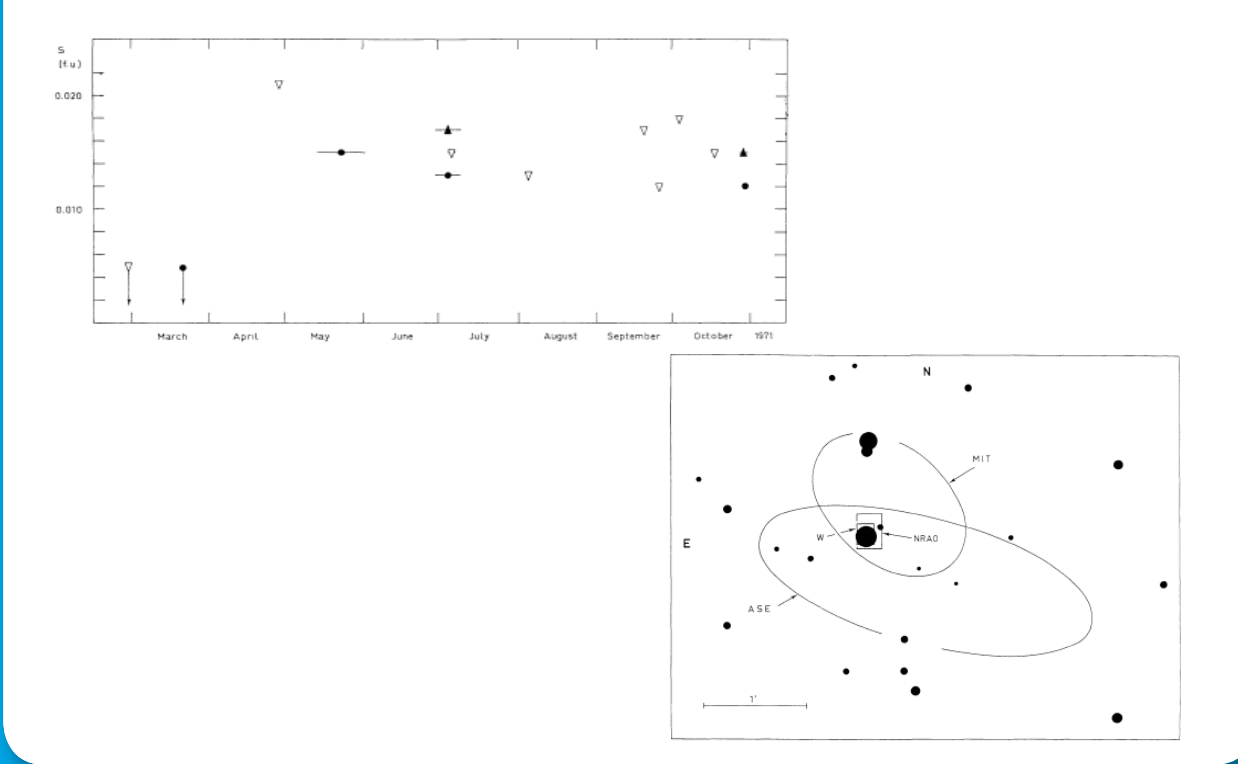

\section{M51 radio spiral arms, 1972}
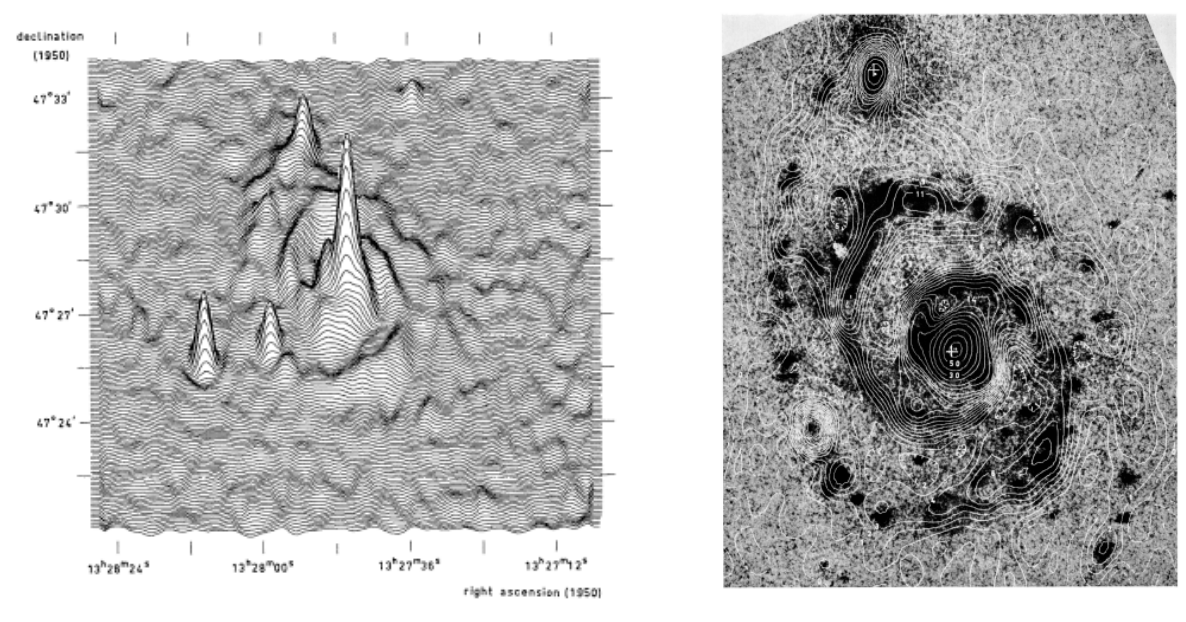

NGC 4258 radio spiral arms, 1972

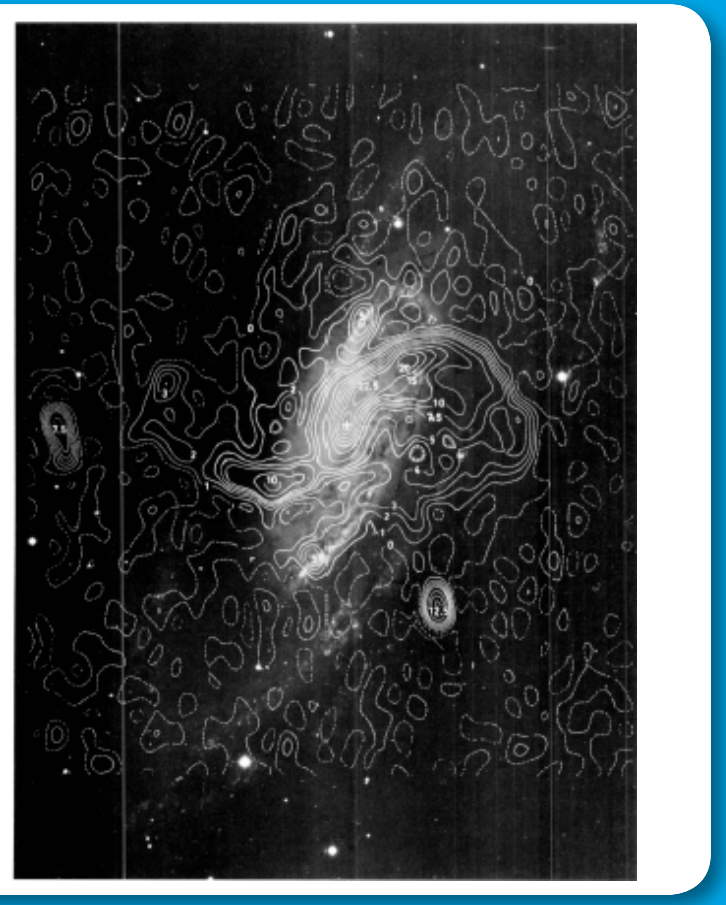

CHAPTER 18 FIFTY WSRT HIGHLIGHTS 


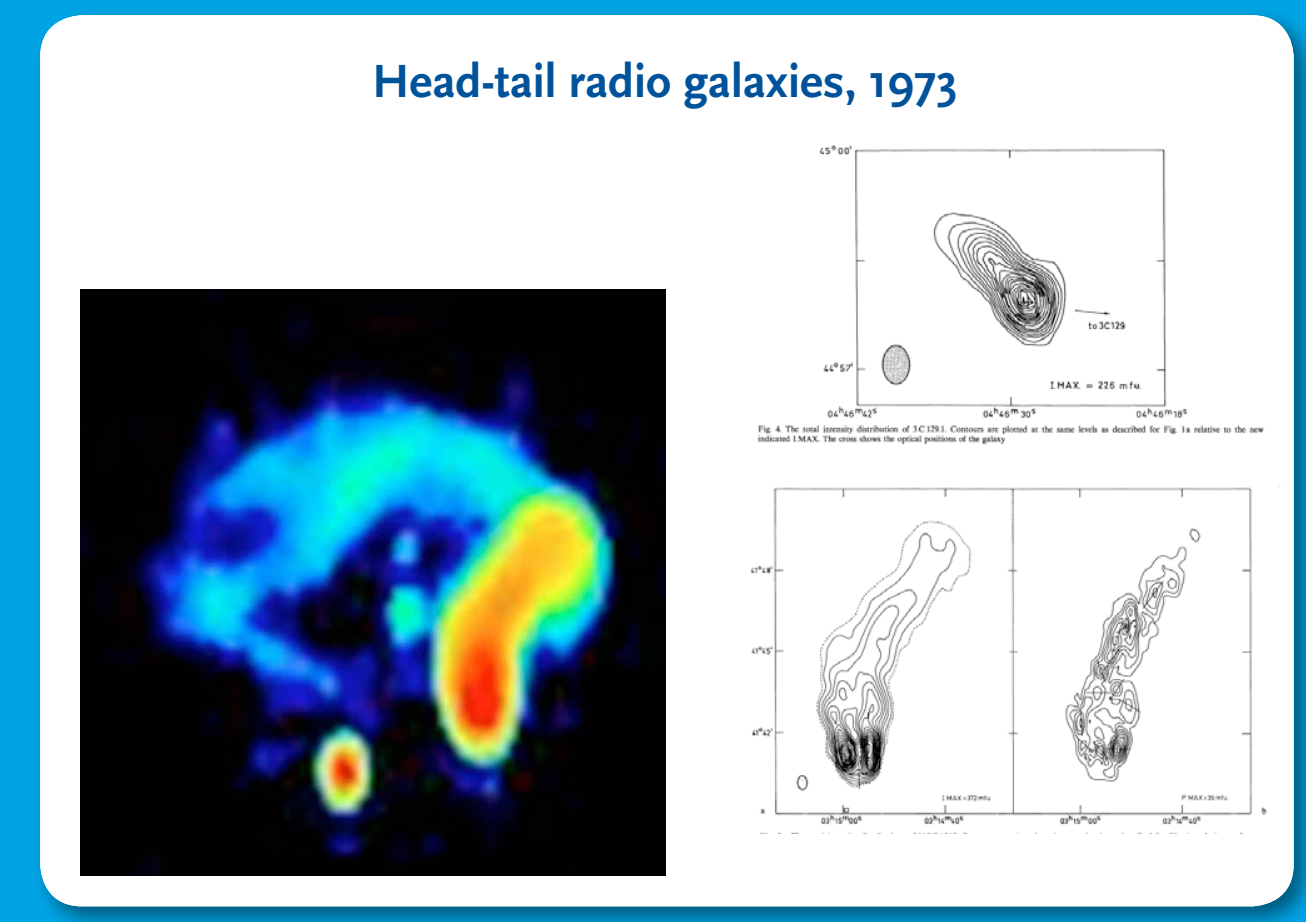

Giant radio galaxies, 1974
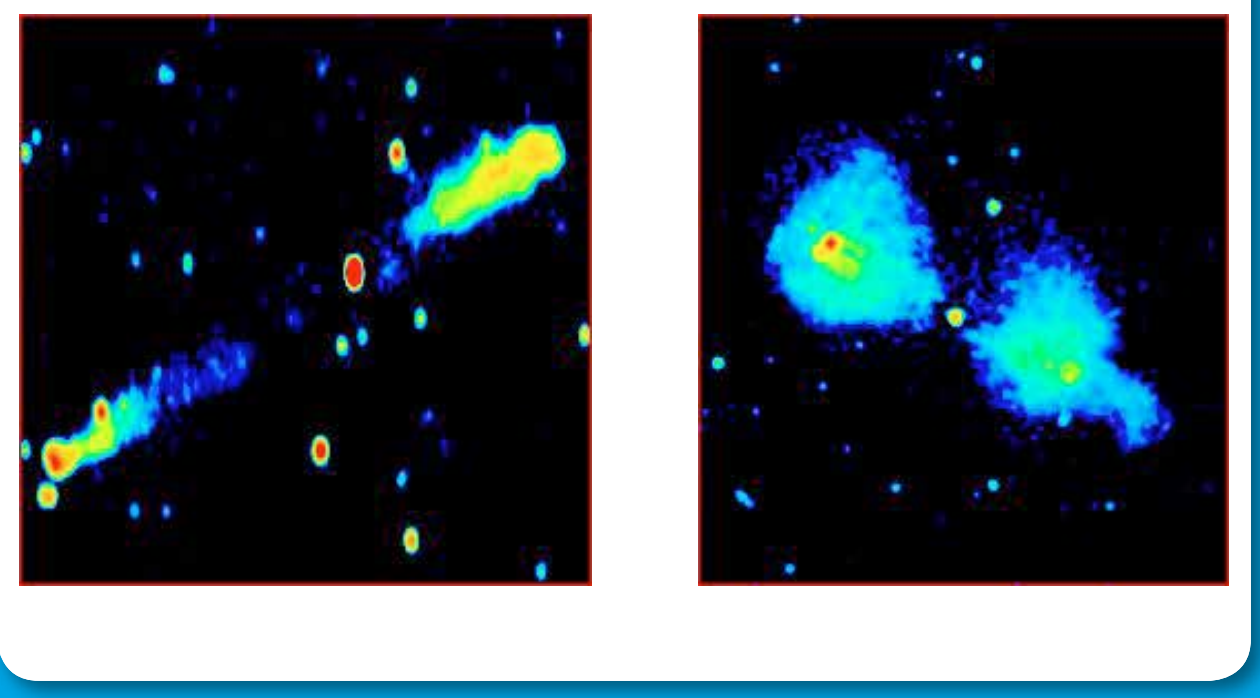

326

50 YEARS WESTERBORK RADIO OBSERVATORY
Högbom CLEAN, 1974

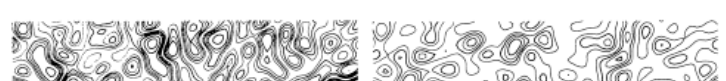

20100\%

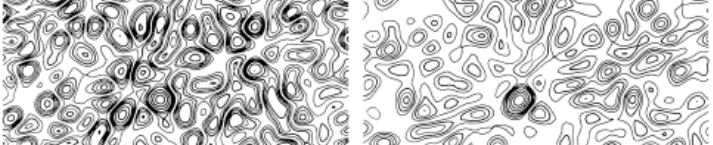

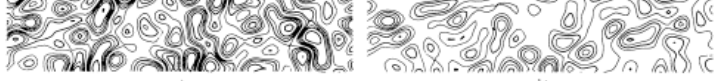

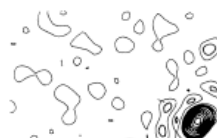

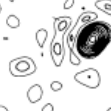

0

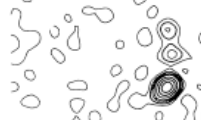

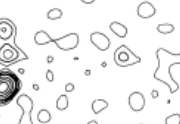

$\therefore$

Hydrogen (HI) in galaxy M81, 1975

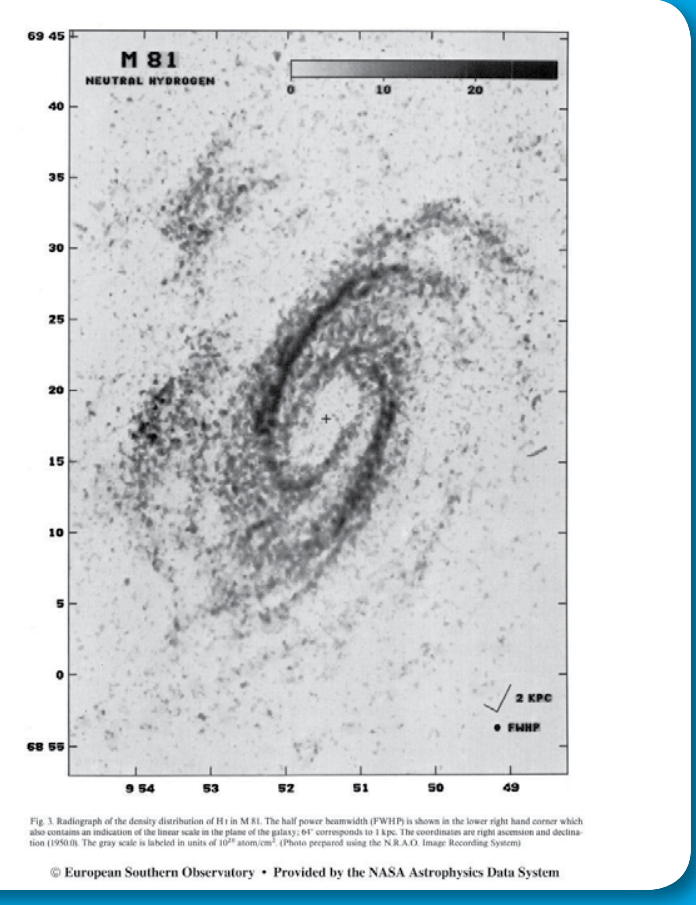

CHAPTER 18 FIFTY WSRT HIGHLIGHTS 

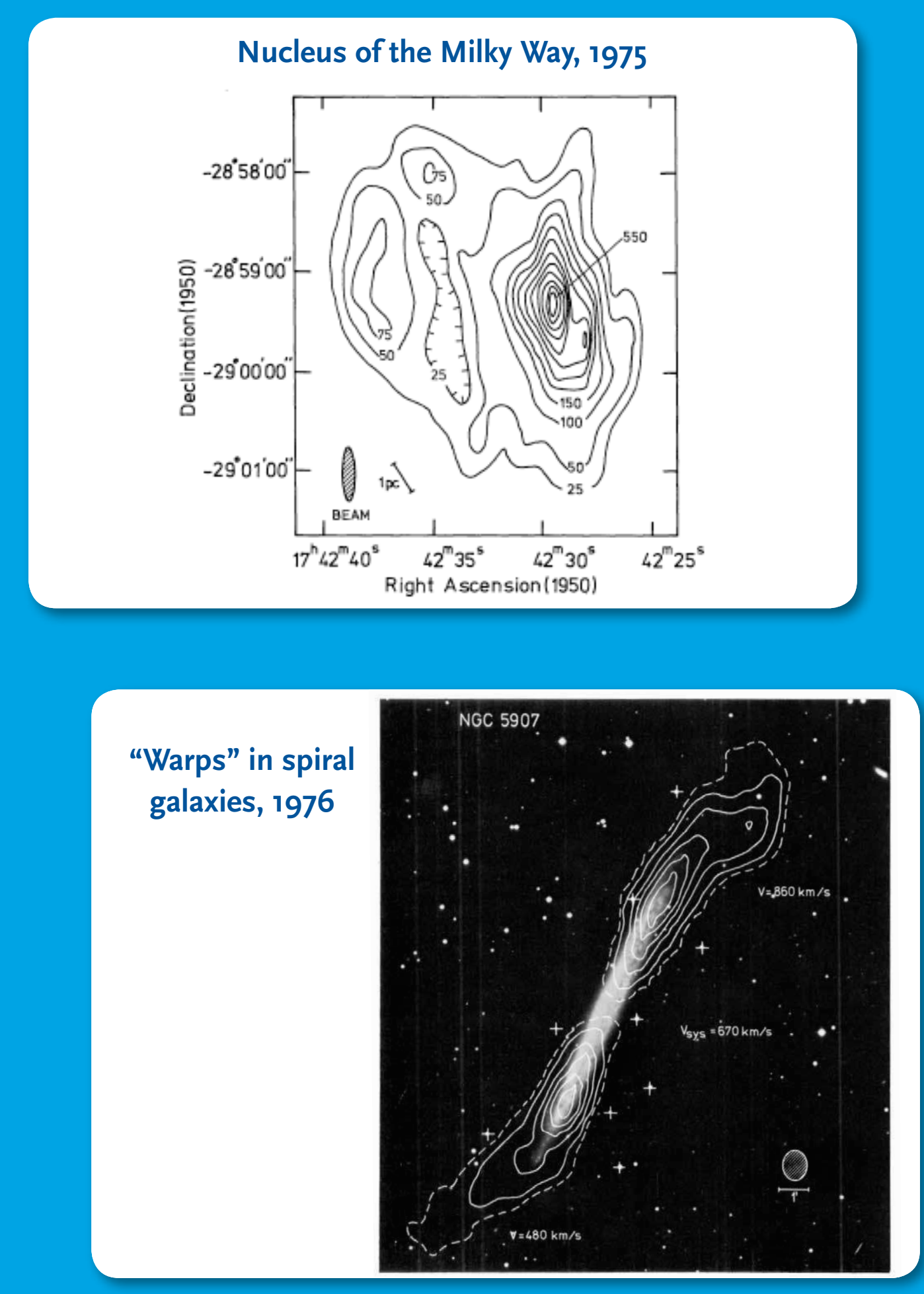

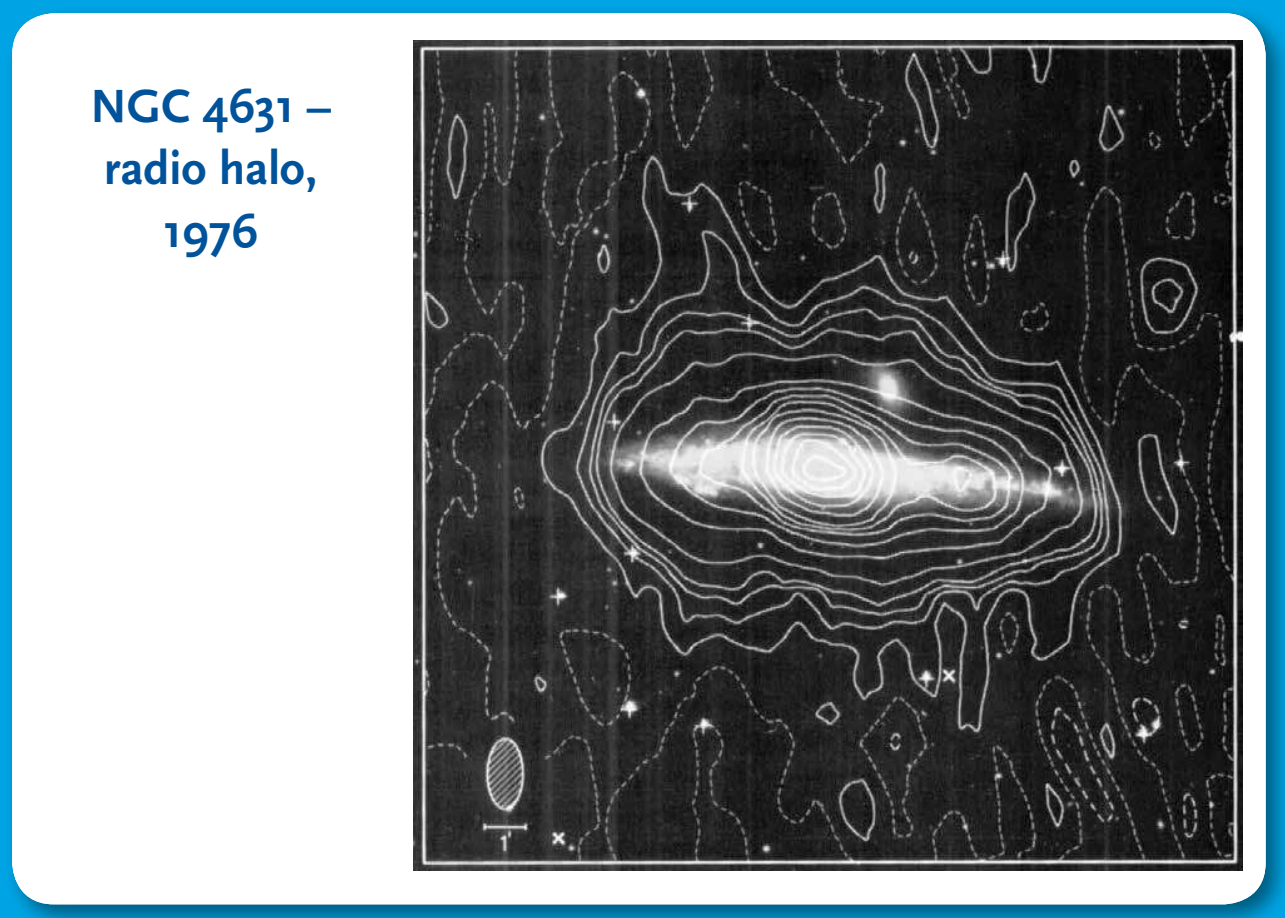

Deep source counts, 1977

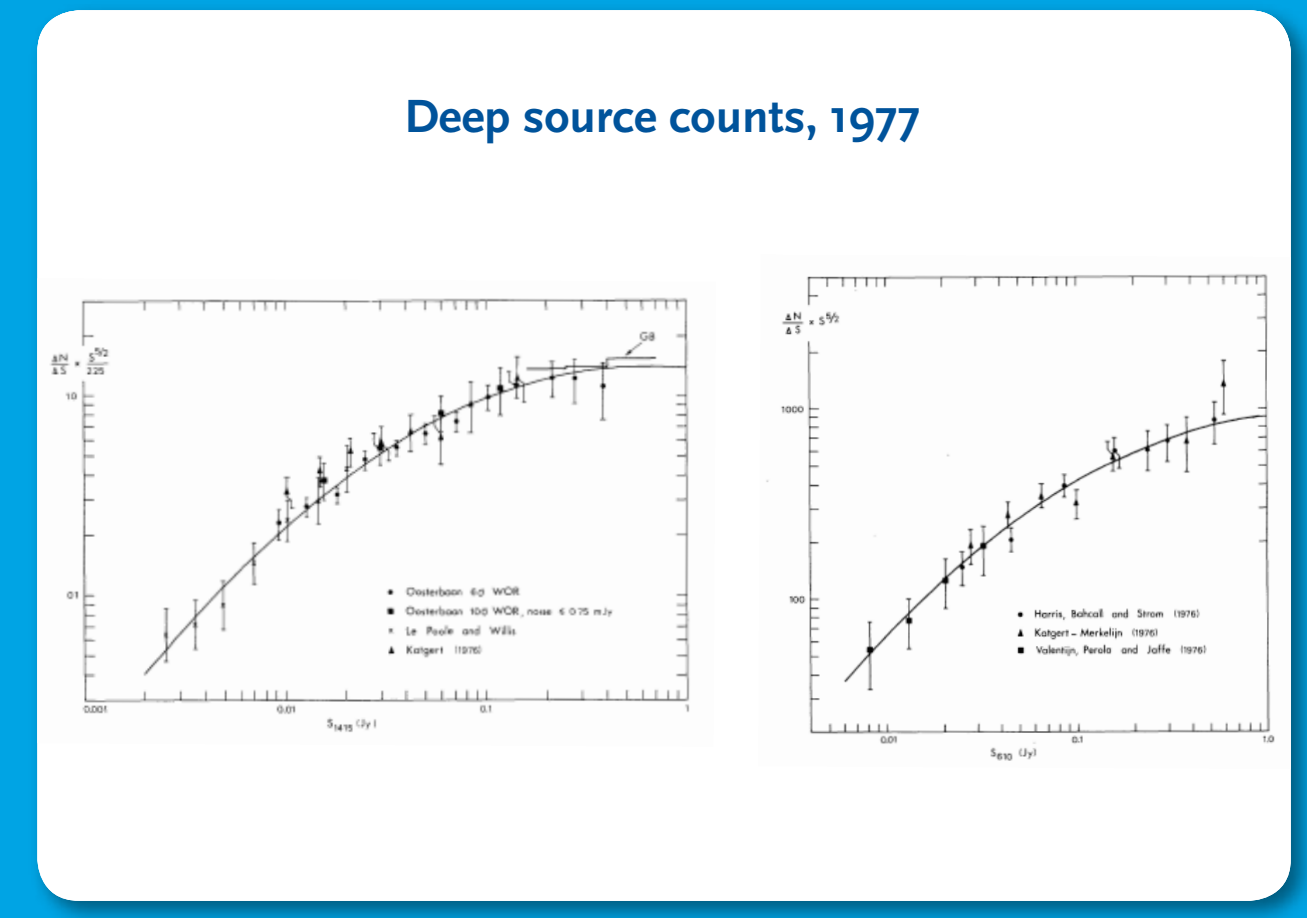

1994

1994
1995
1996

1996

1997

1998

1999

2000

2001

2002

2003

2004

2005

2006

2007

2008

2009

2010

2011

2012 


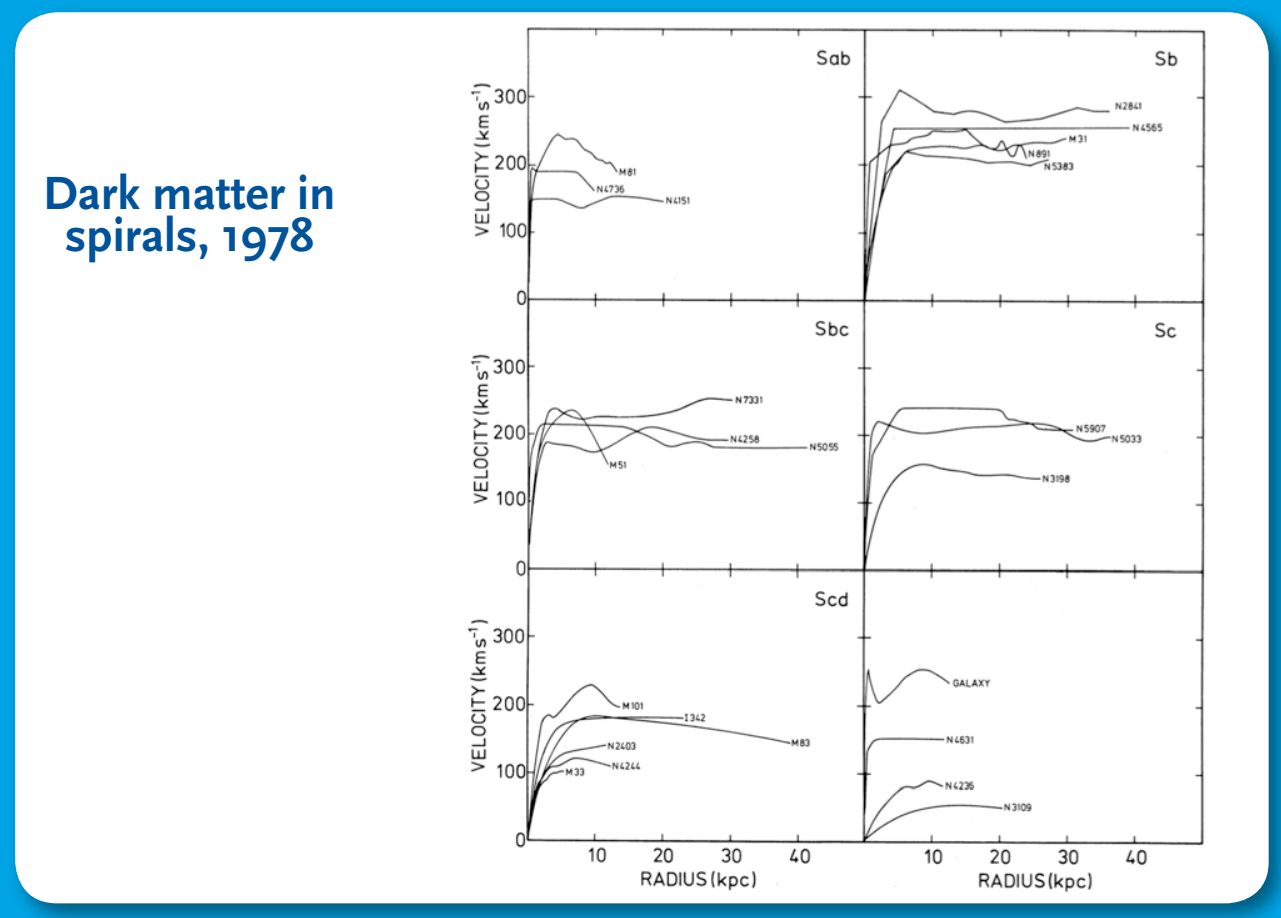

\section{NGC $891 \mathrm{HI}$, a milky way edge-on, 1979}

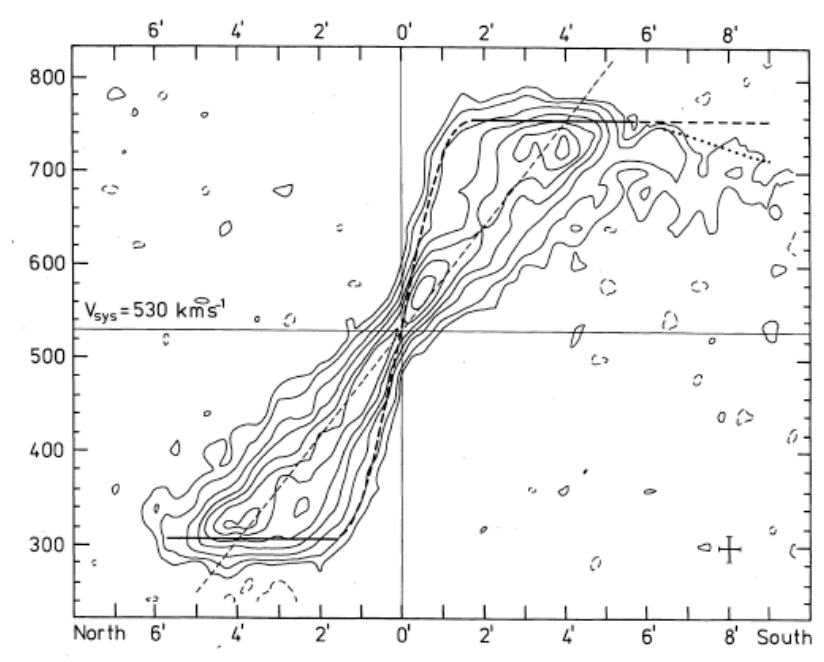

$\underbrace{2}_{0.0}$
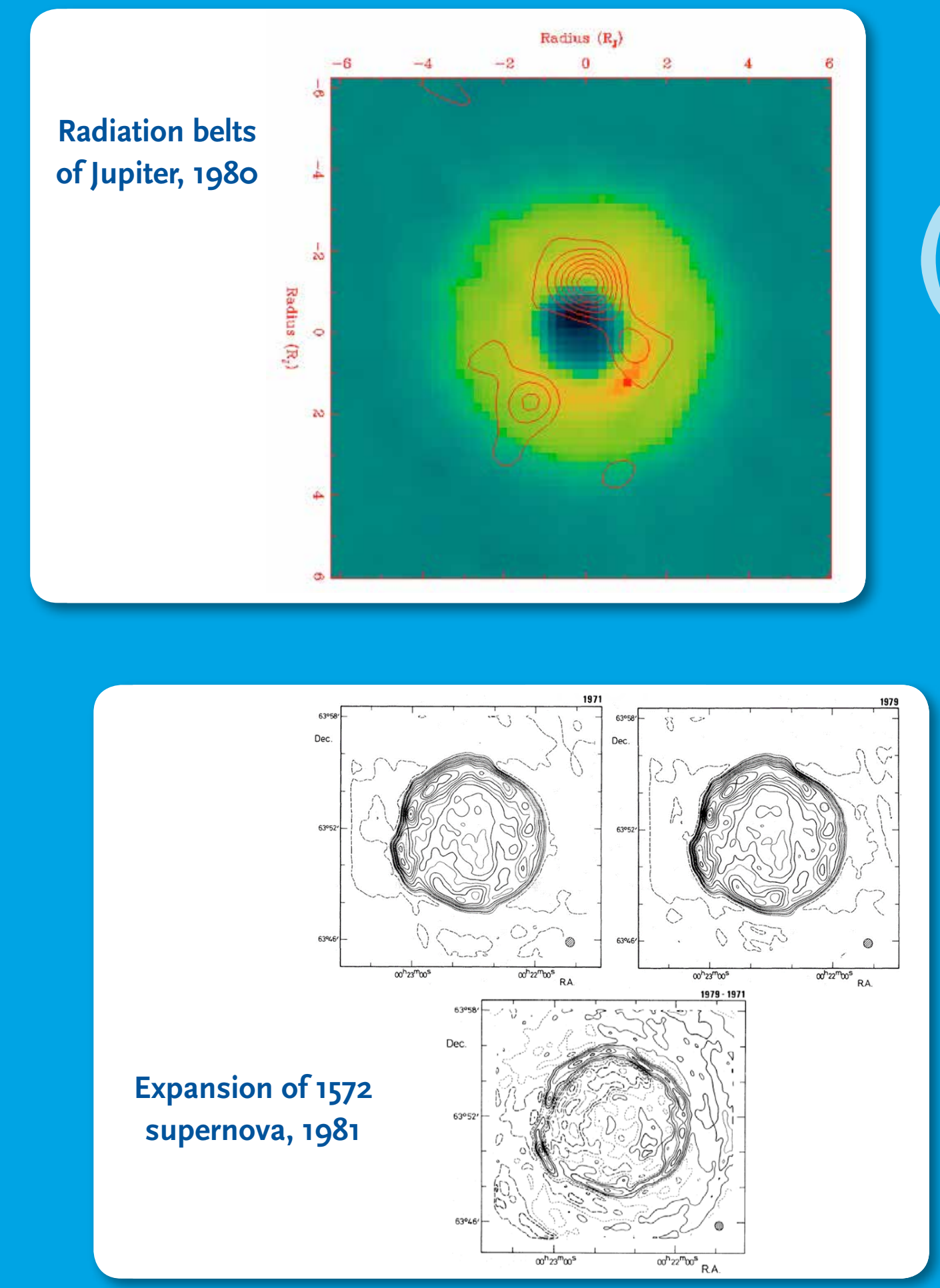
ms pulsar PSR 1937+214 in 4C21.53, 1982
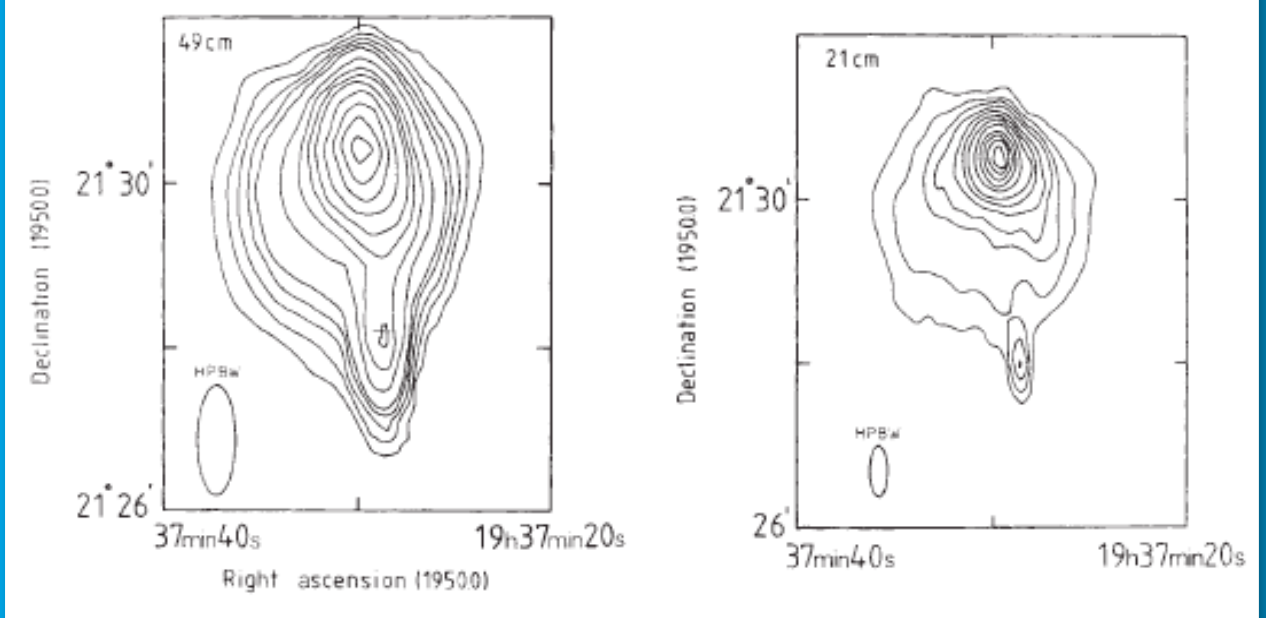

Redundancy: High Dynamic

Range imaging of the

Perseus cluster, 1982

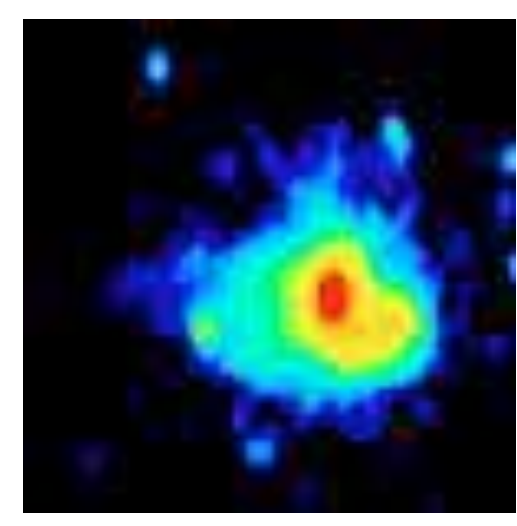

50 YEARS WESTERBORK RADIO OBSERVATORY

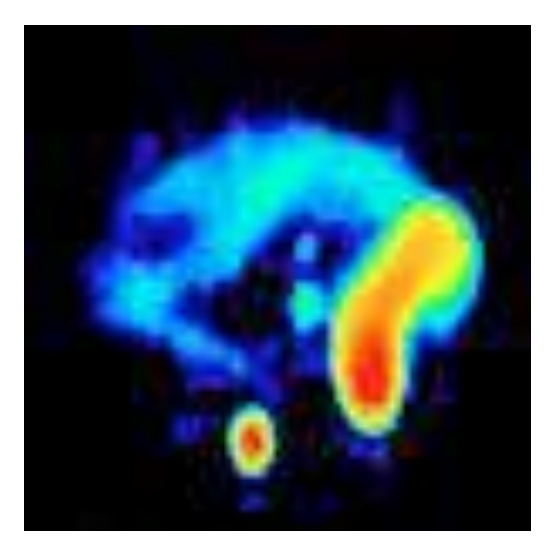

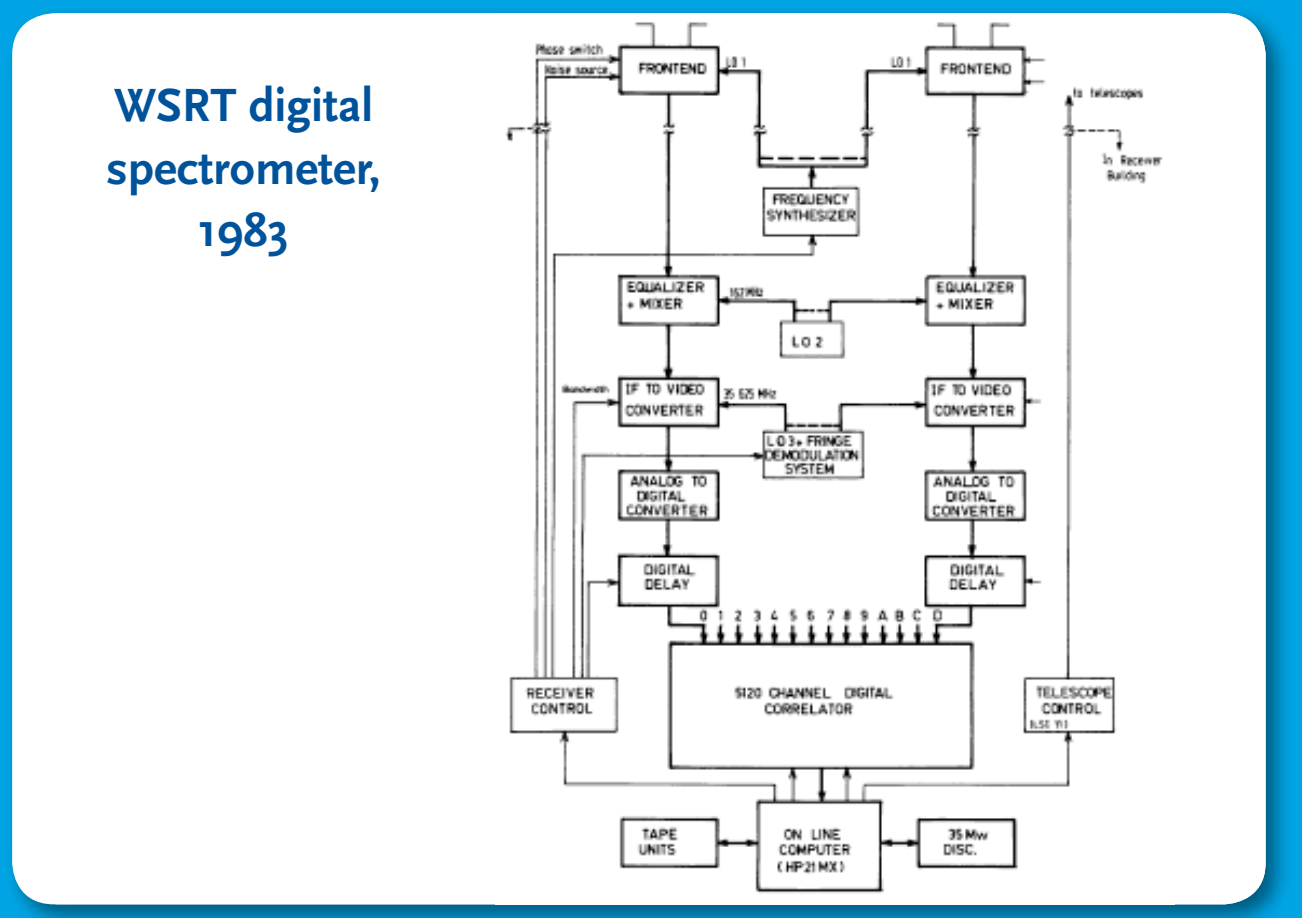

\section{Deep continuum surveys, 1984}
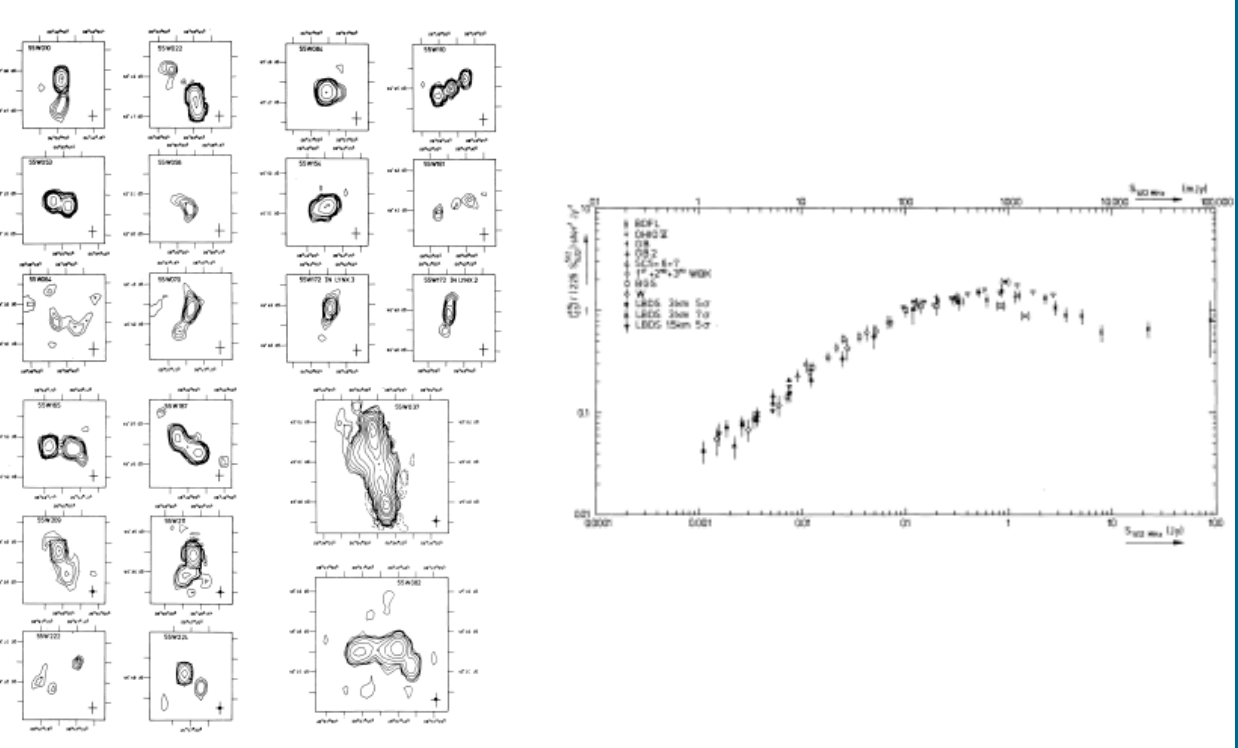

1994

1995

1996

1997

1998

1999

2000

2001

2002

2003

2004

2005

2006

2007

2008

2009

2010

2011

2012 


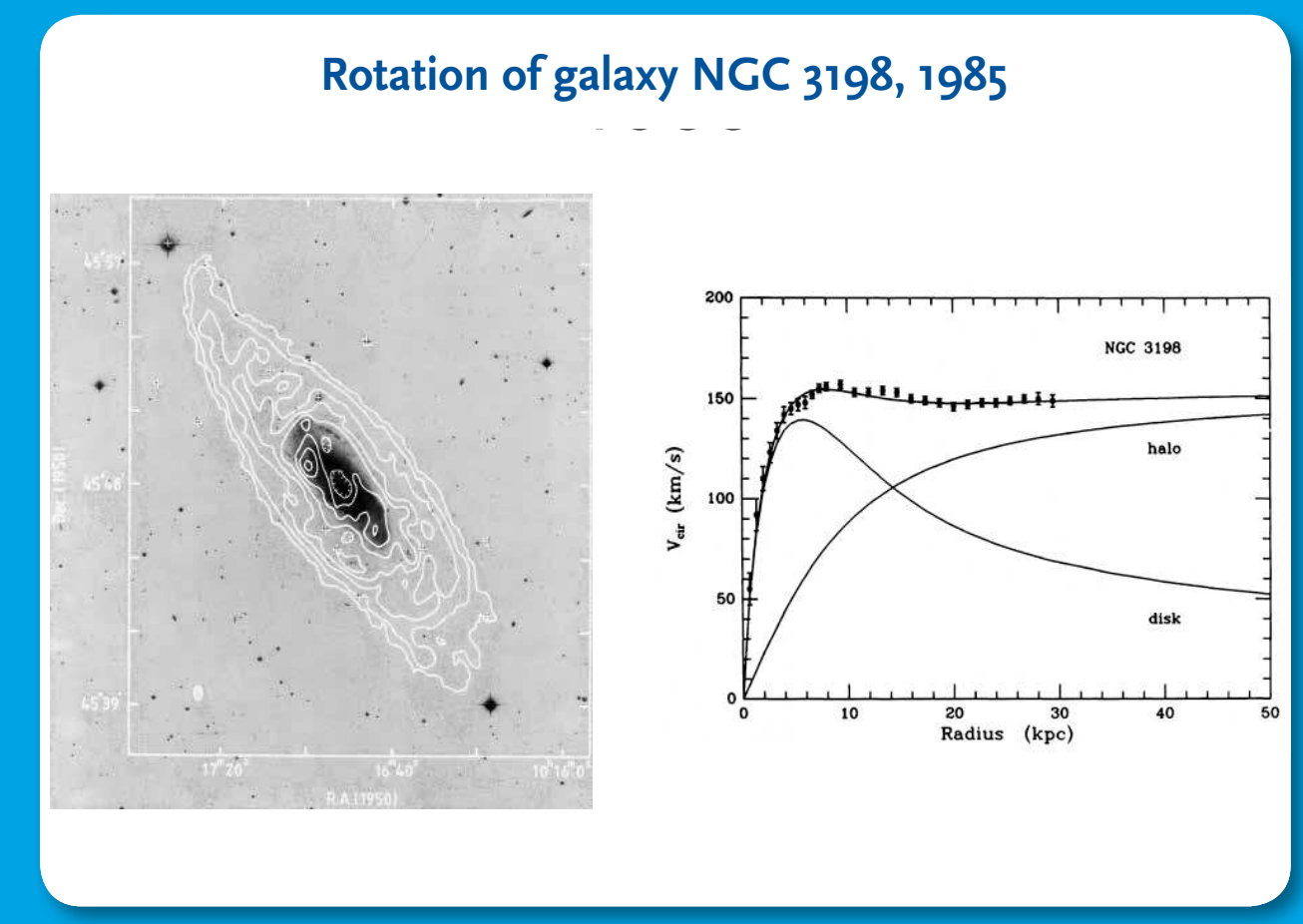

Radio galaxy polarization, 1986
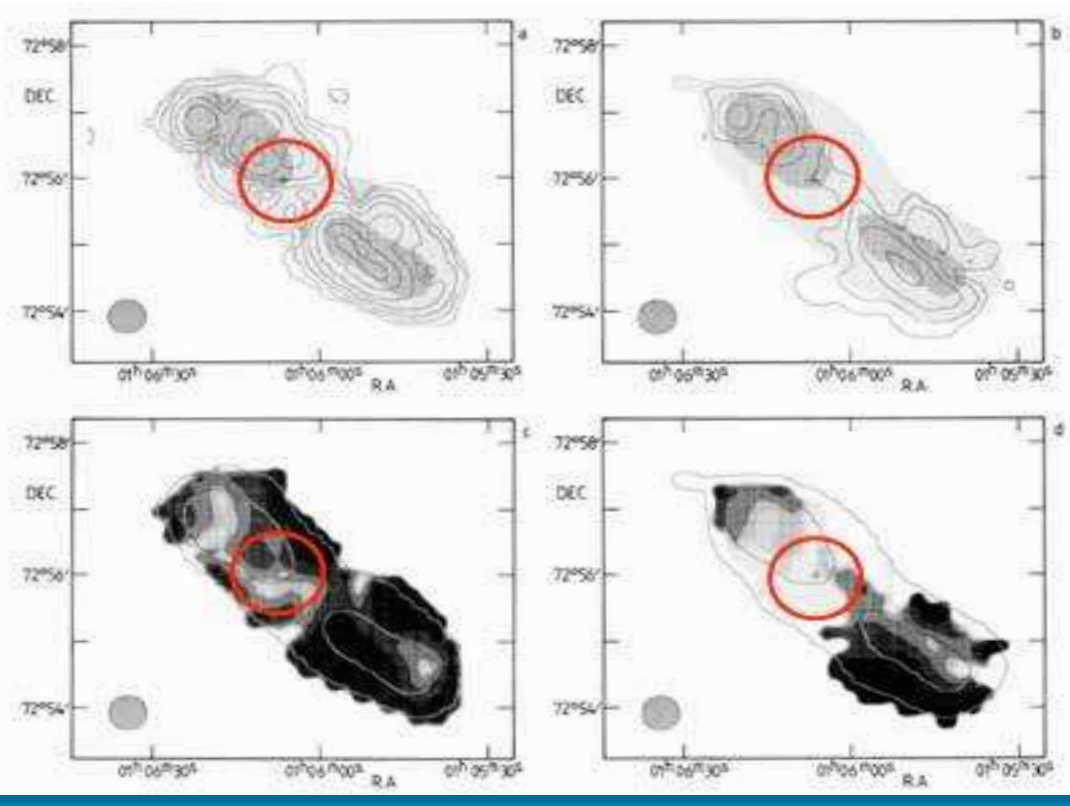

Deep Source Counts, 1987

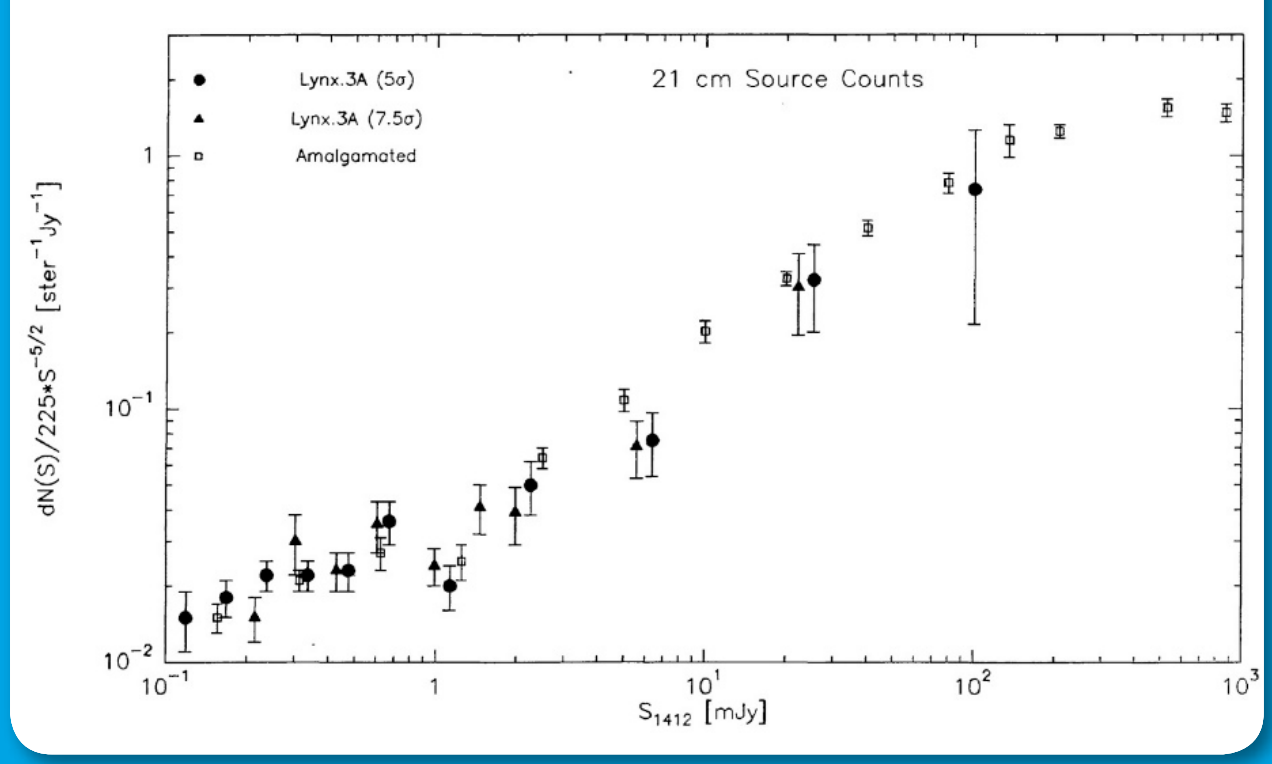

High velocity clouds - M101, 1988

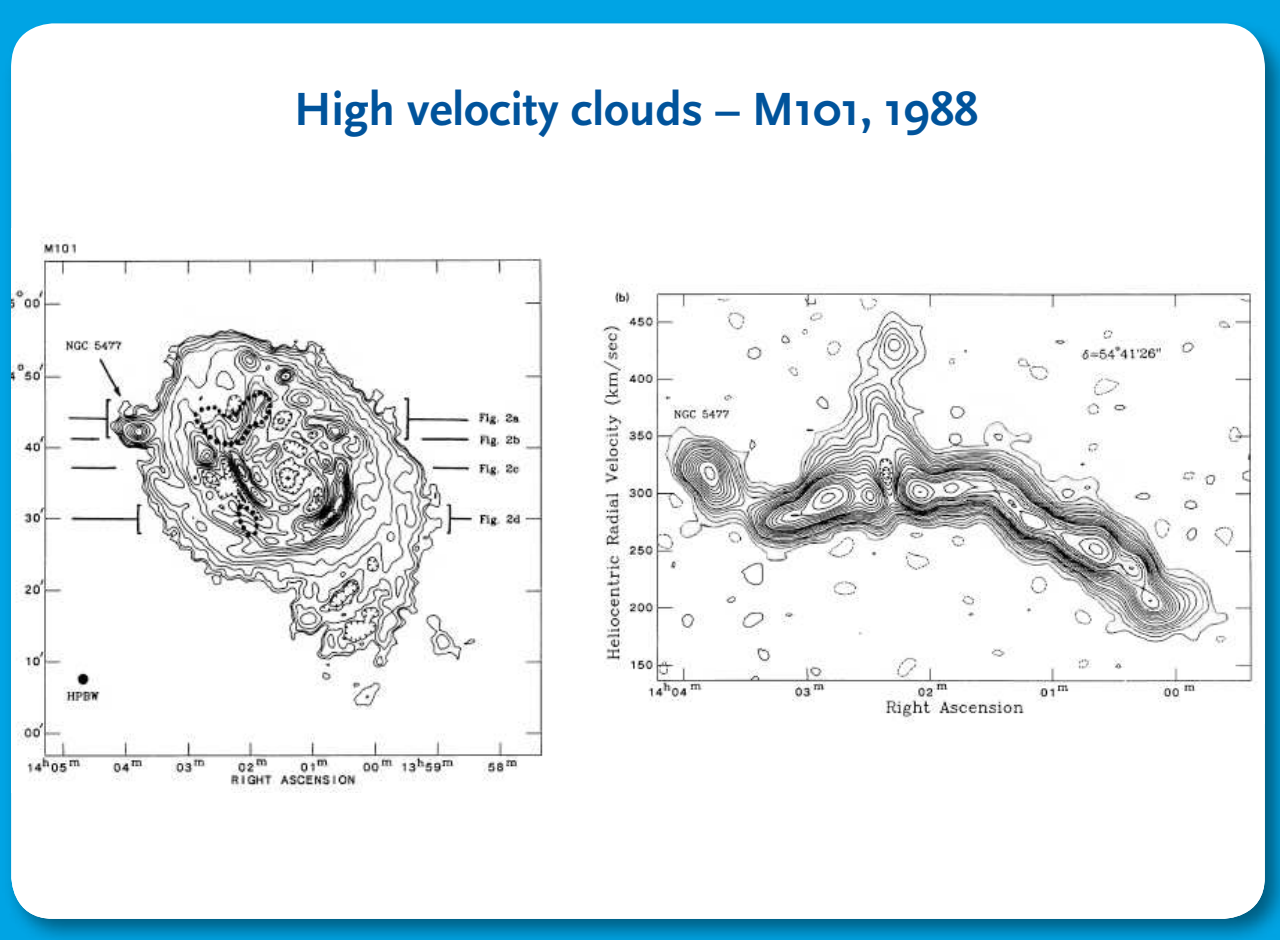

CHAPTER 18 FIFTY WSRT HIGHLIGHTS 
No Hydrogen “pancakes”, 1992

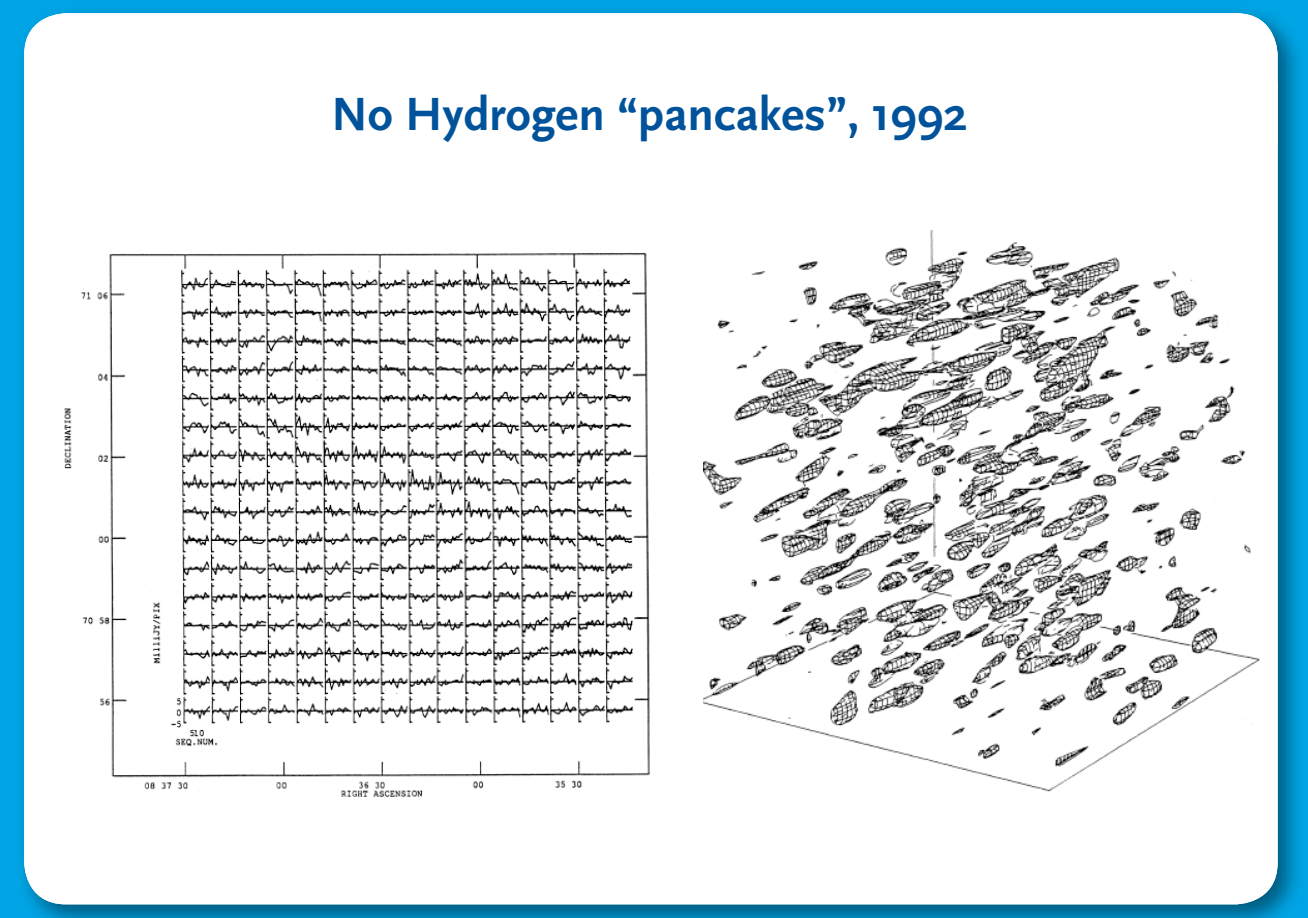

Milky Way high velocity clouds, 1991

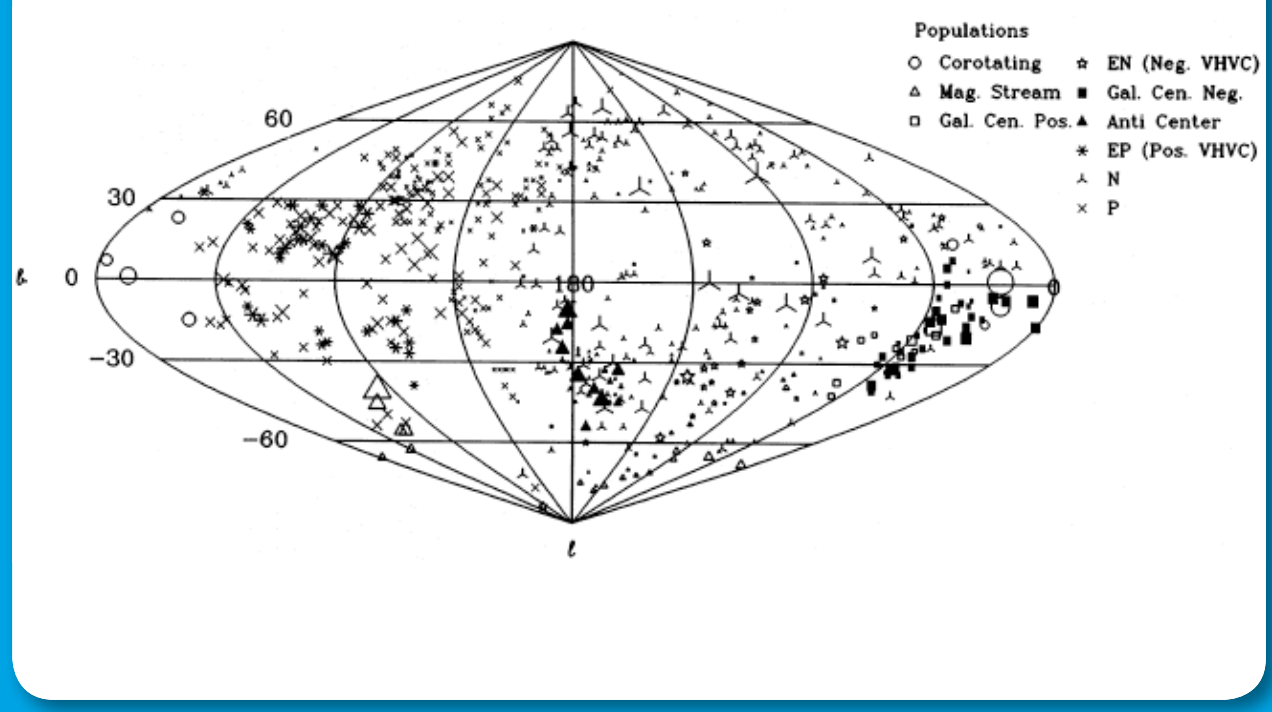

Netherlands East West Synthesis Telescope Array Reduction

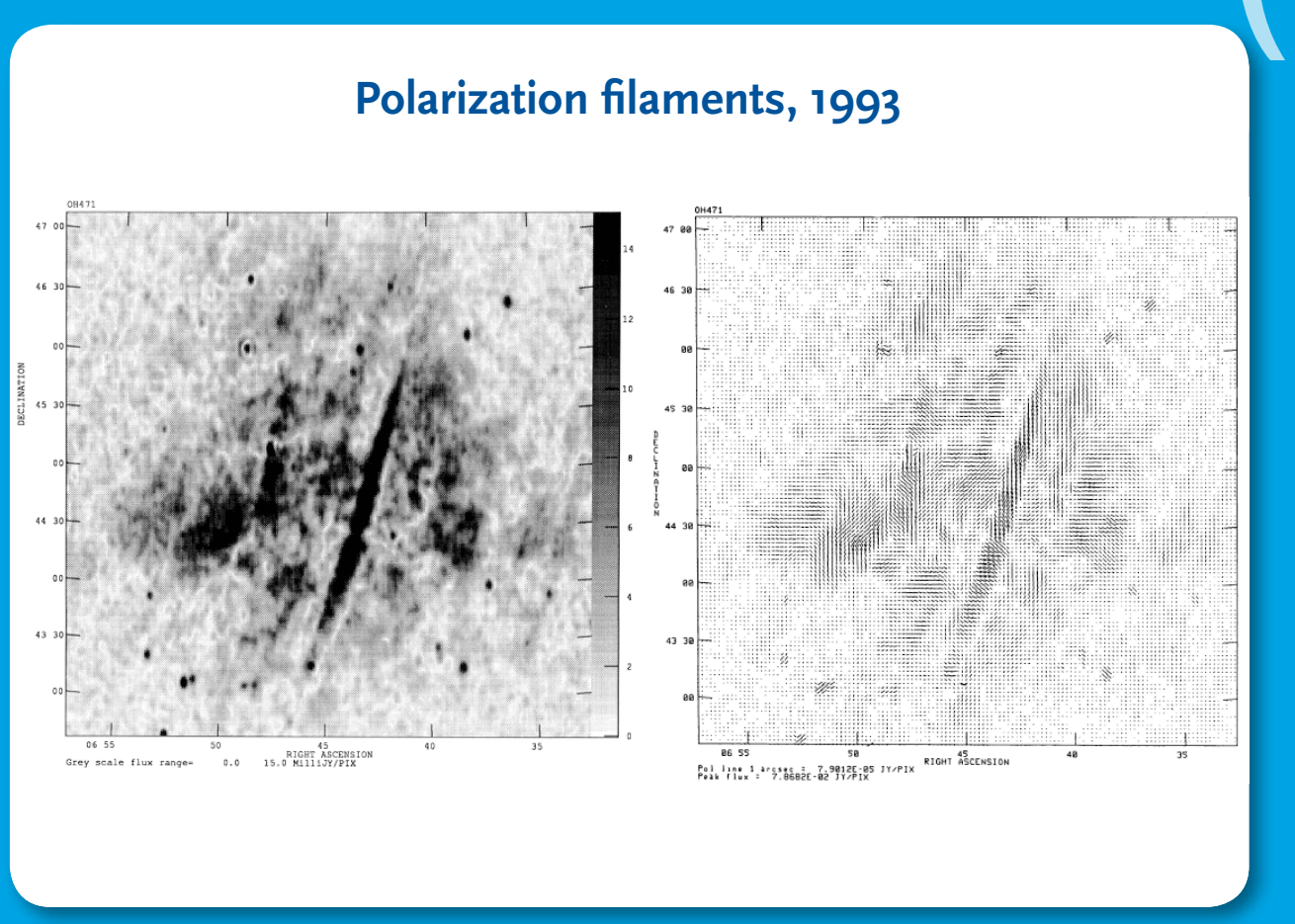

CHAPTER 18 FIFTY WSRT HIGHLIGHTS 

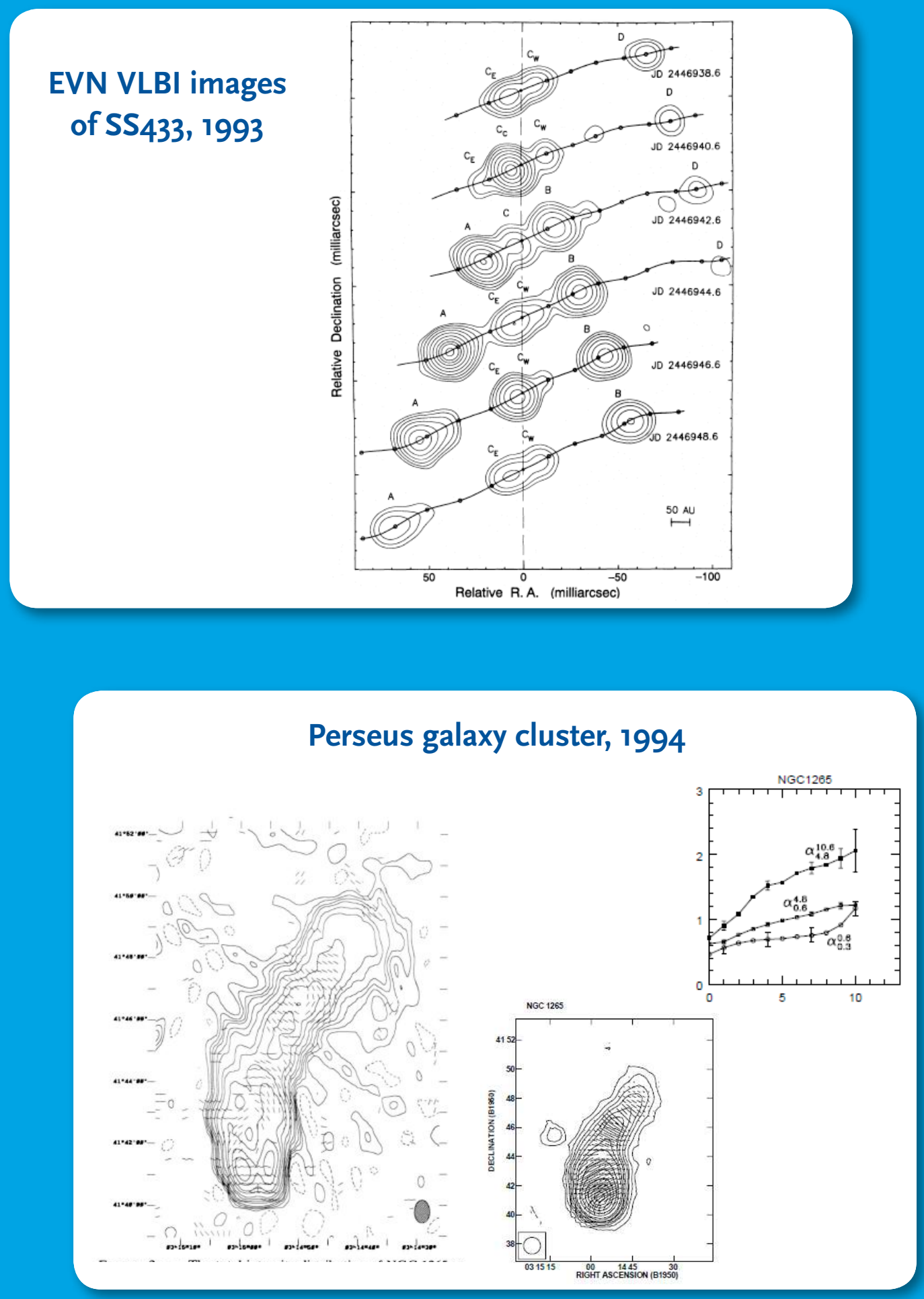
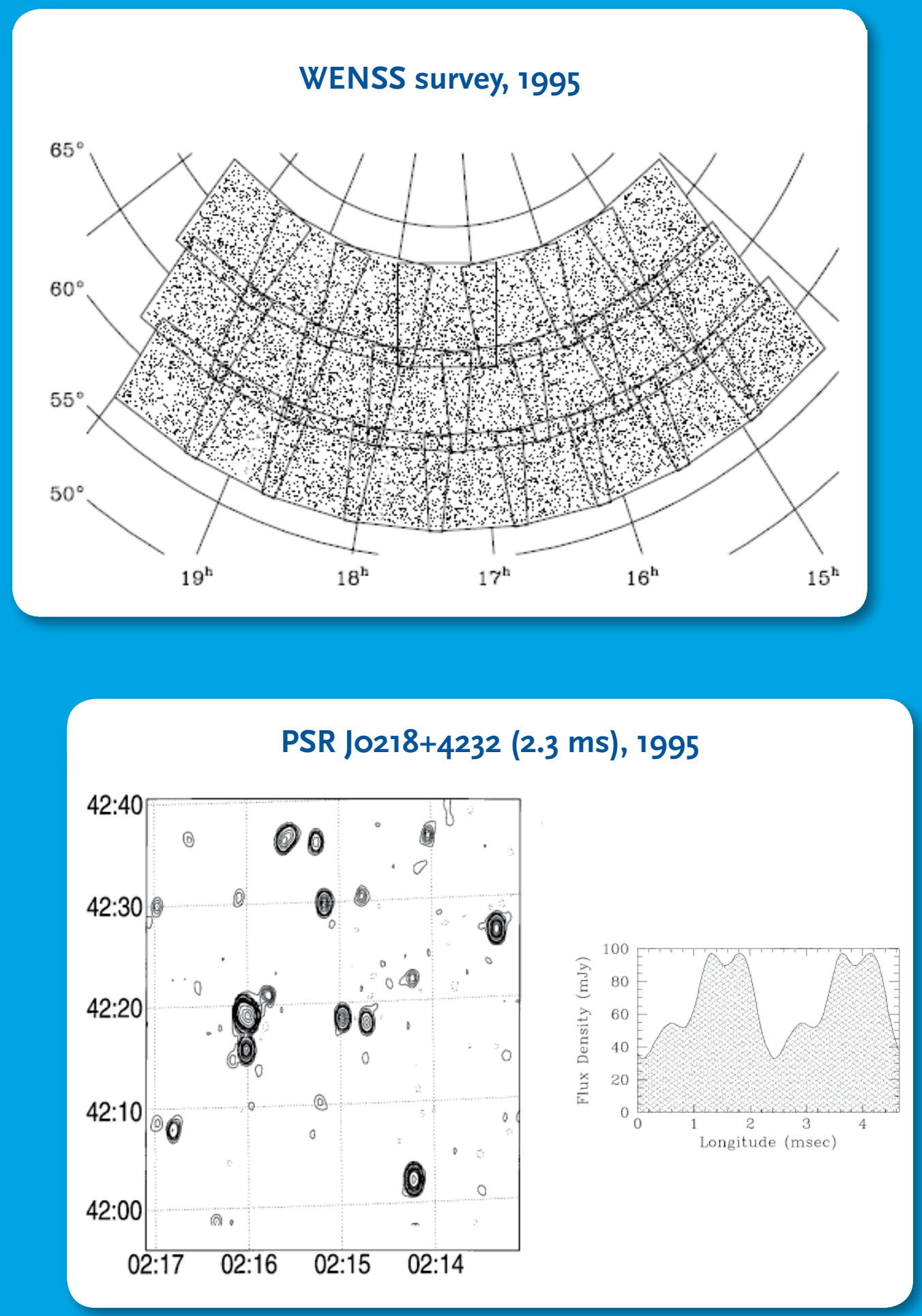

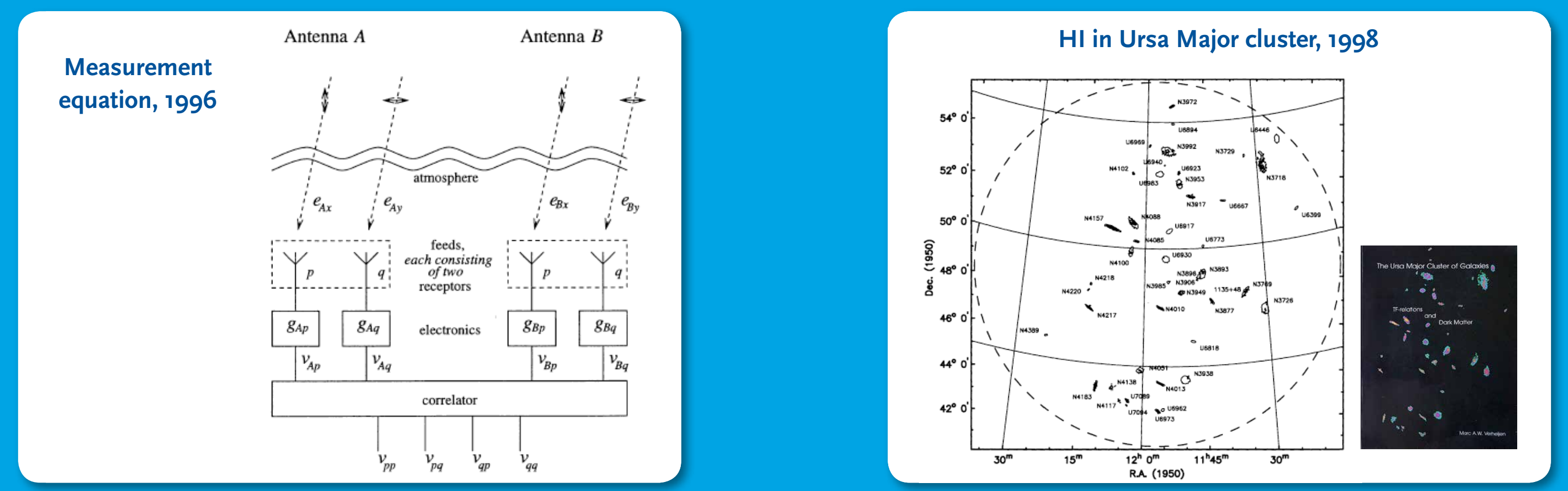

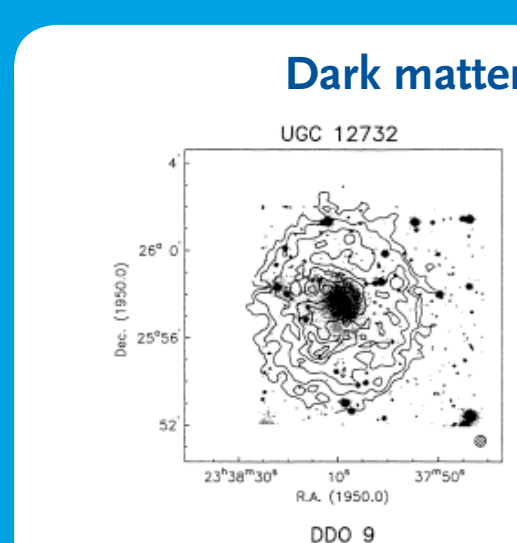

DDO 9
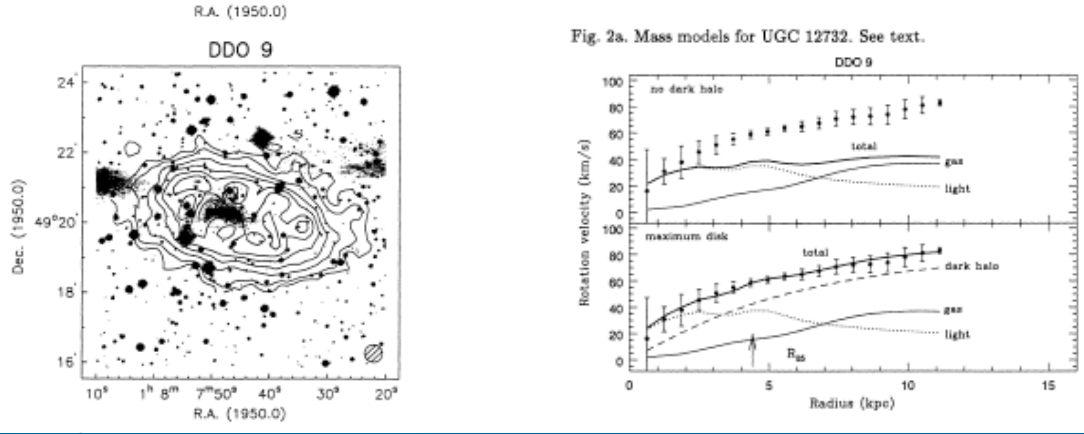

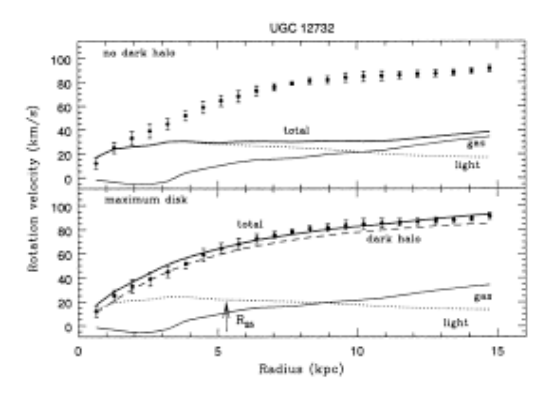

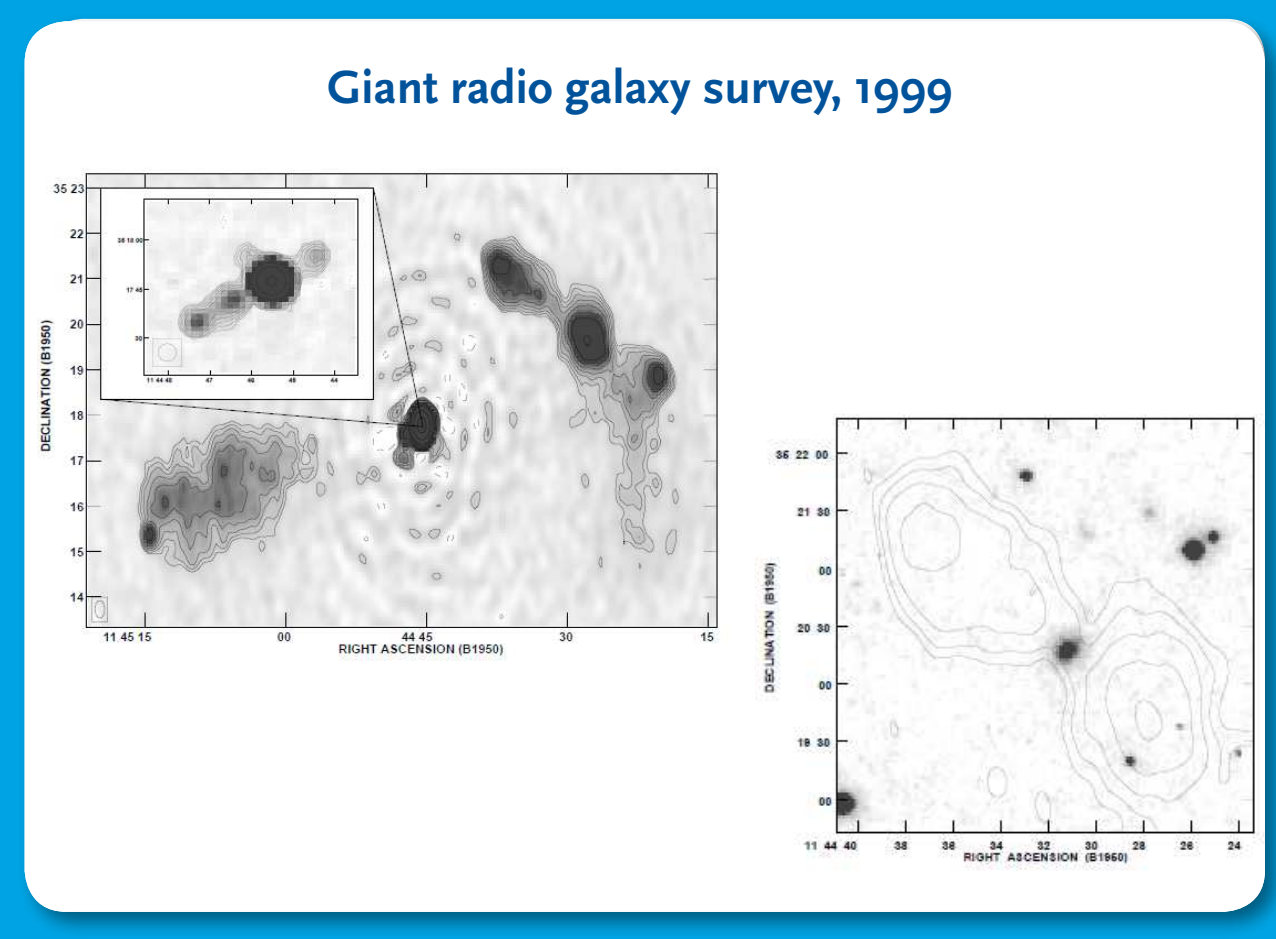

CHAPTER 18 FIFTY WSRT HIGHLIGHTS 
Hubble deep-field images,

2000

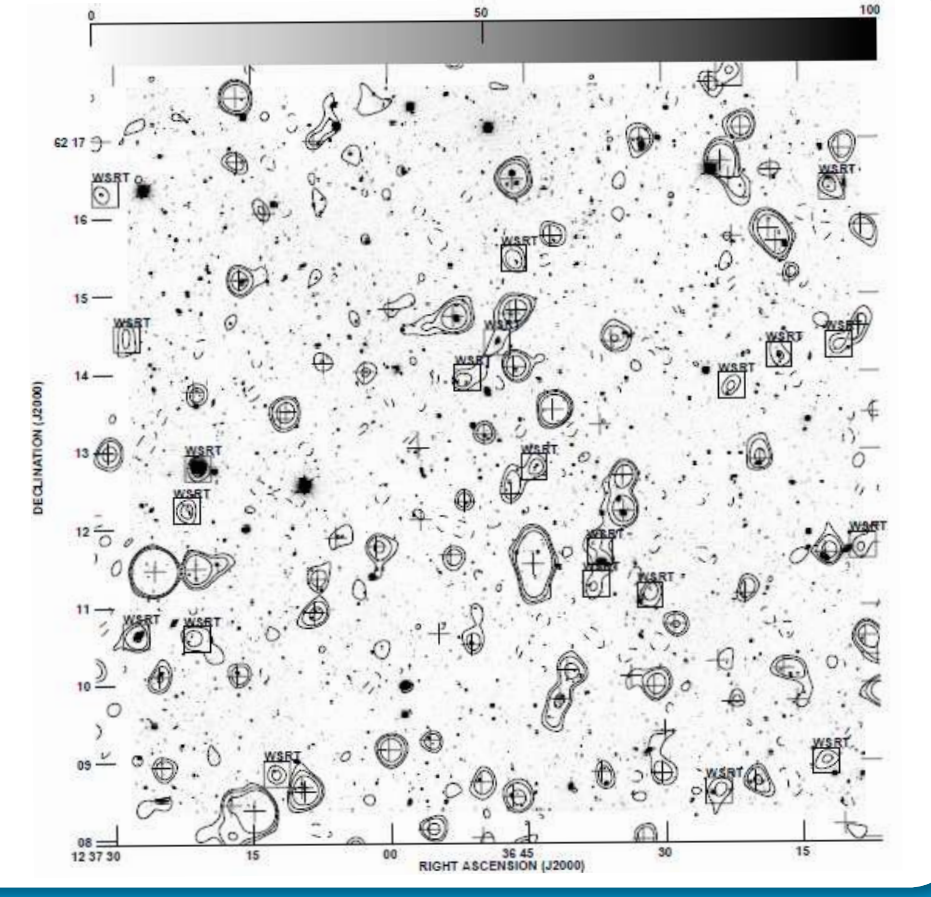

WESTERBORK 2000

after the system upgrade

Upgraded Telescope Receivers Signalconditioning Central processing

$\frac{\text { Receivers }}{(1 \text { per telescope) }} \frac{\text { Signalconditioning }}{(14 \text { telescopes }}$
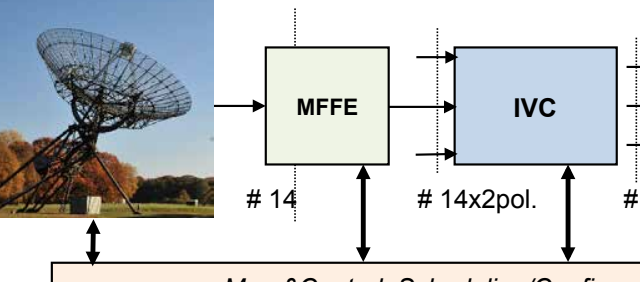

Mon.\&Control, Scheduling/C

TMS

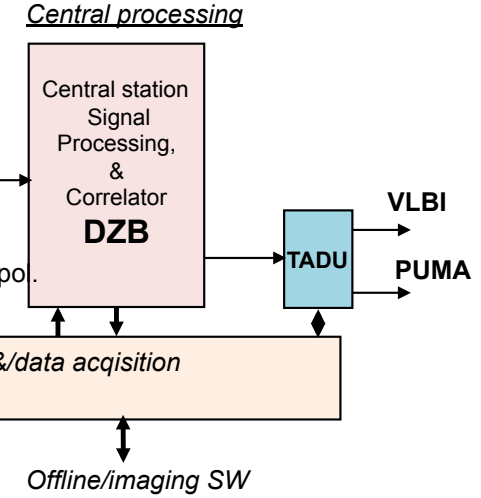

Variable quasar

J1819+3845, 2001

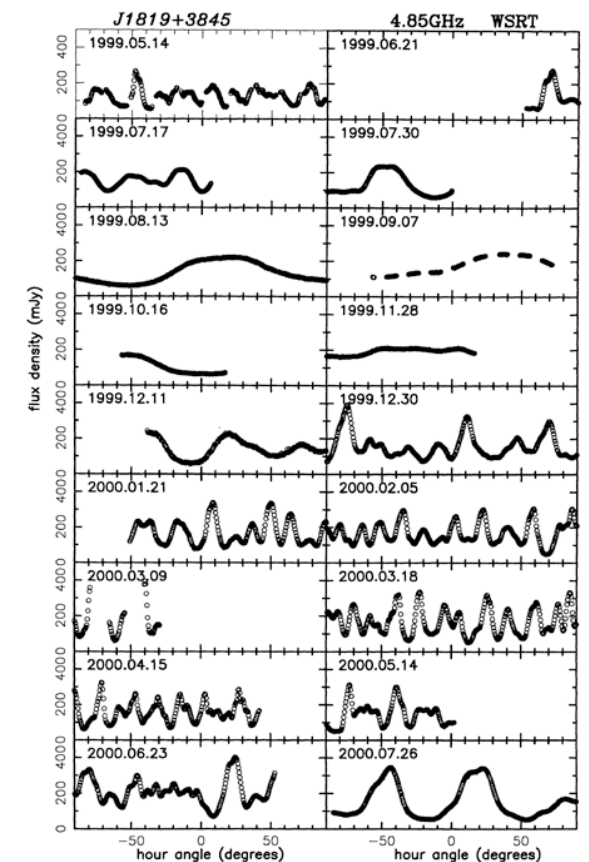

WHISP HI galaxy survey, 2002 Overview UGC 7353
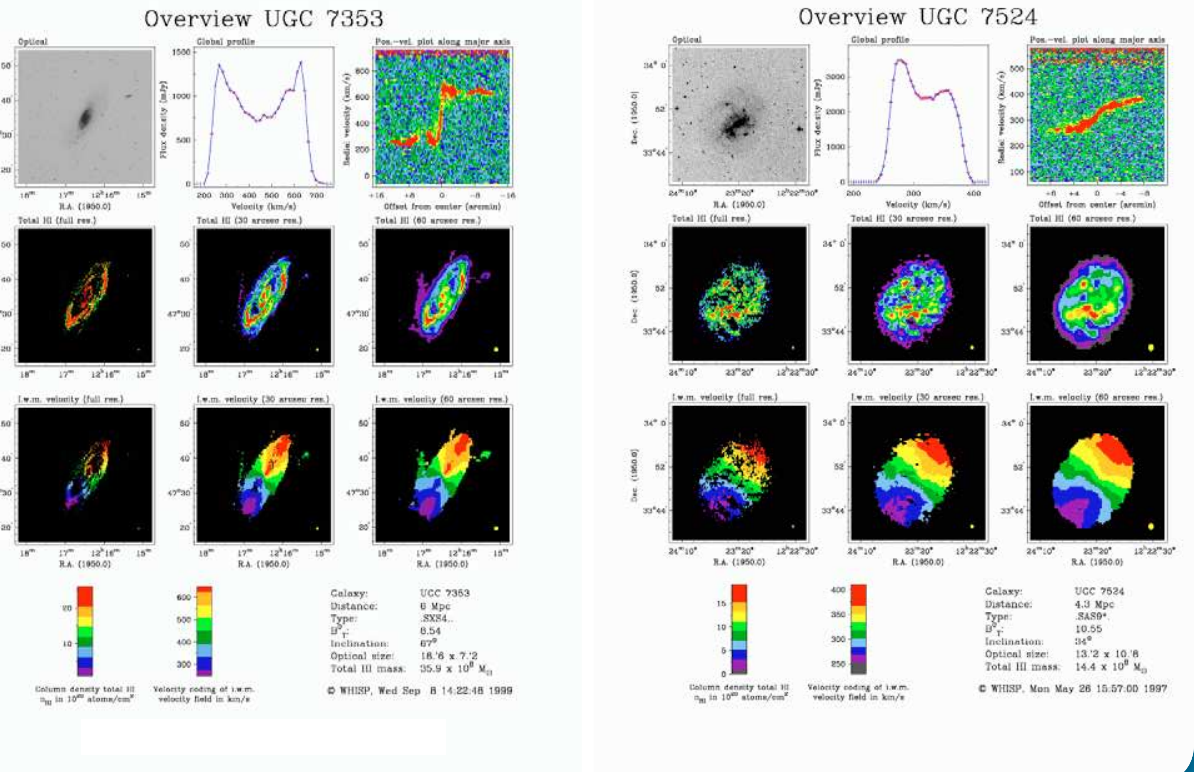

CHAPTER 18 FIFTY WSRT HIGHLIGHTS 

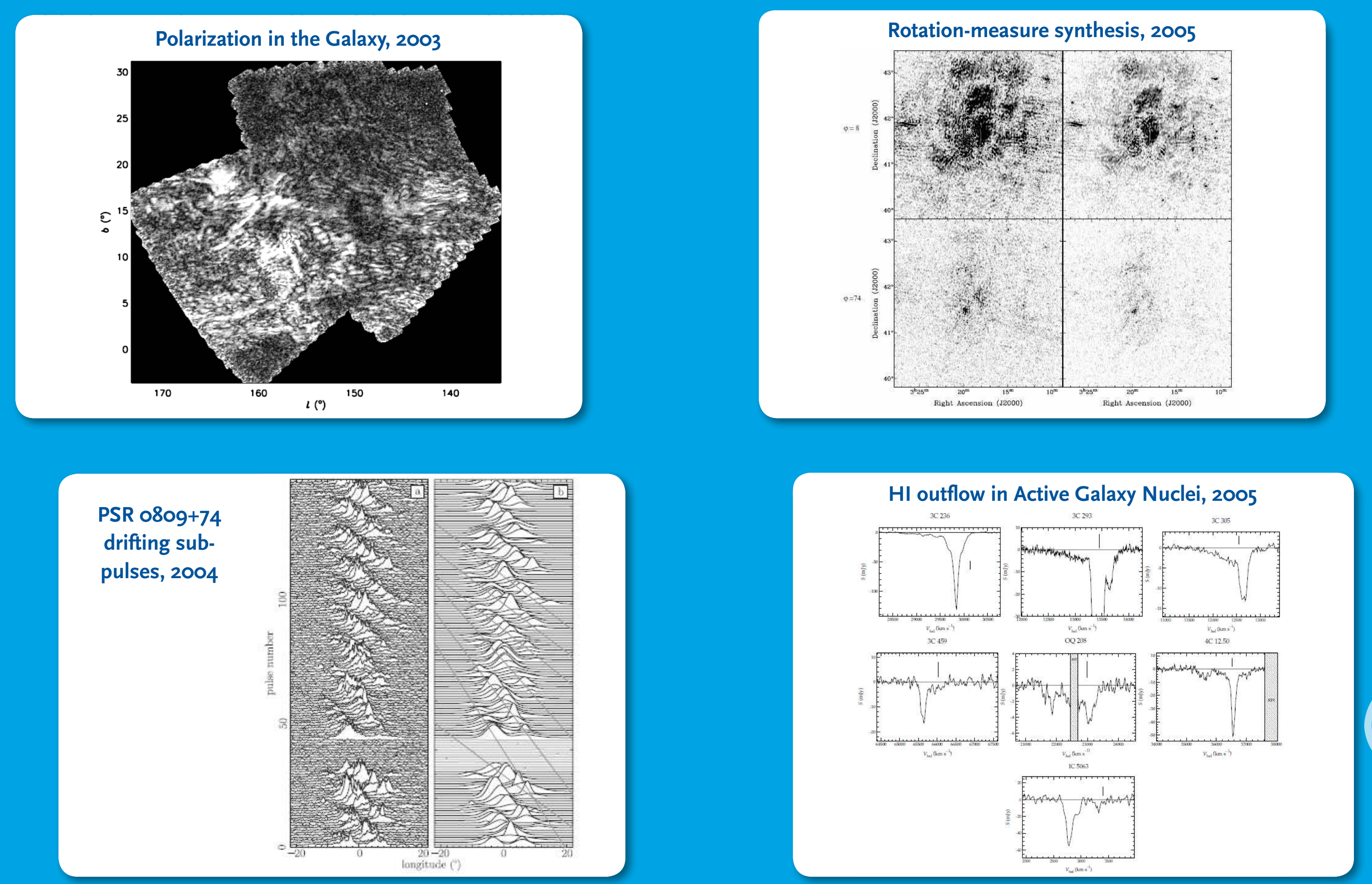

1992

1993

1994

1995

1996

1997

1998

1999

2000

2001

2002

2003

2004

2005

2006

2007

2008

2009

2010

2011

2012 

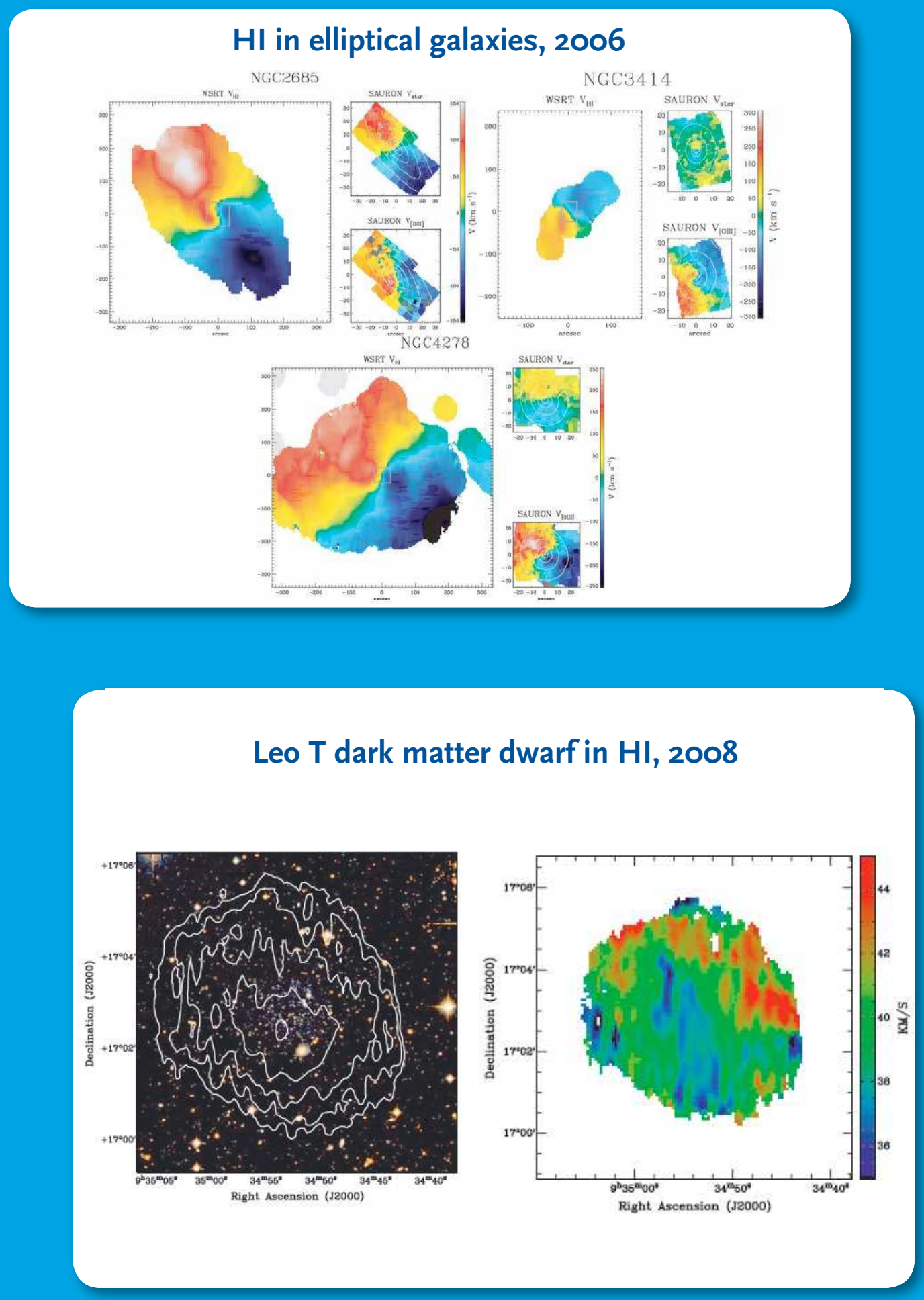
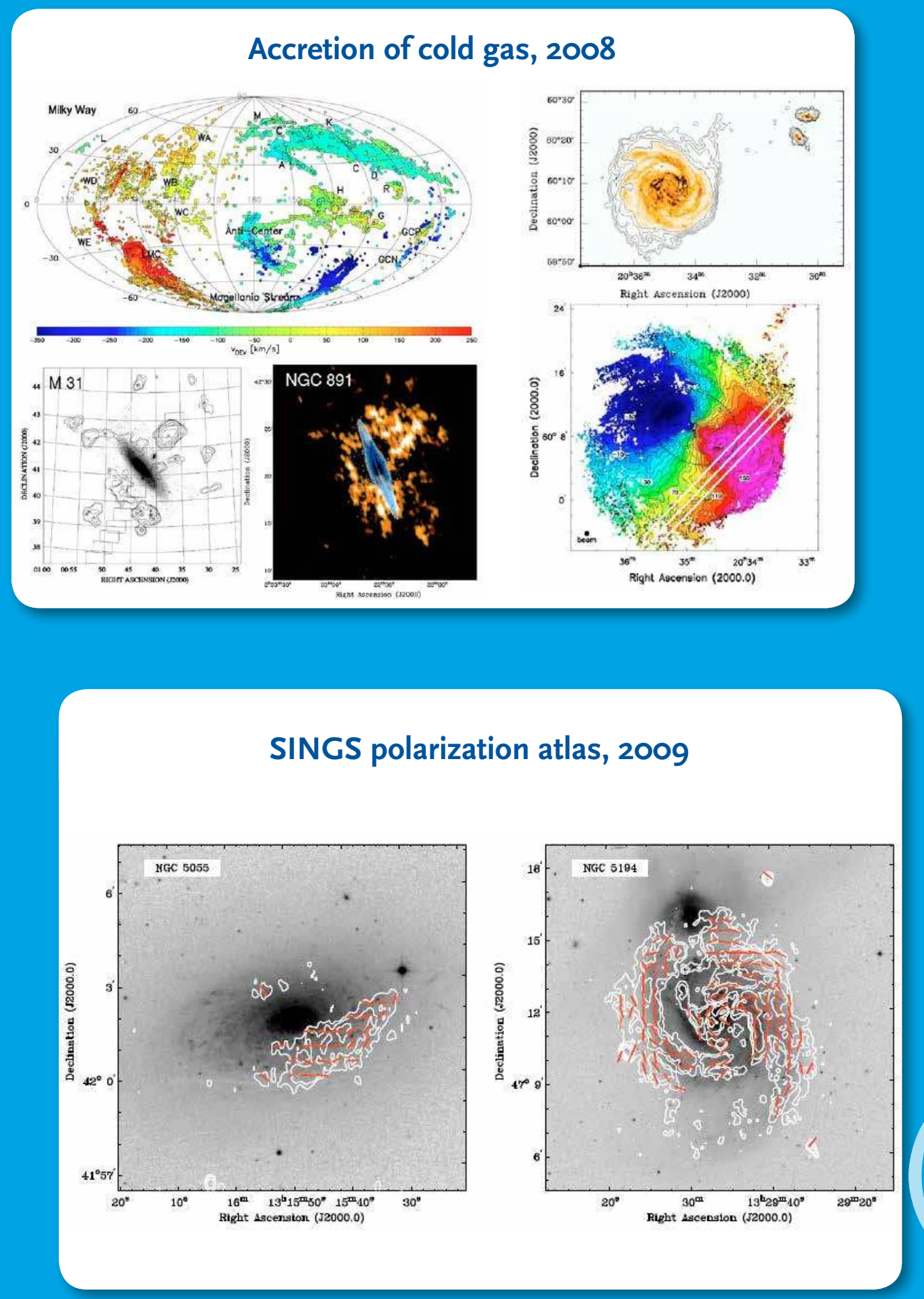

CHAPTER 18 FIFTY WSRT HIGHLIGHTS 

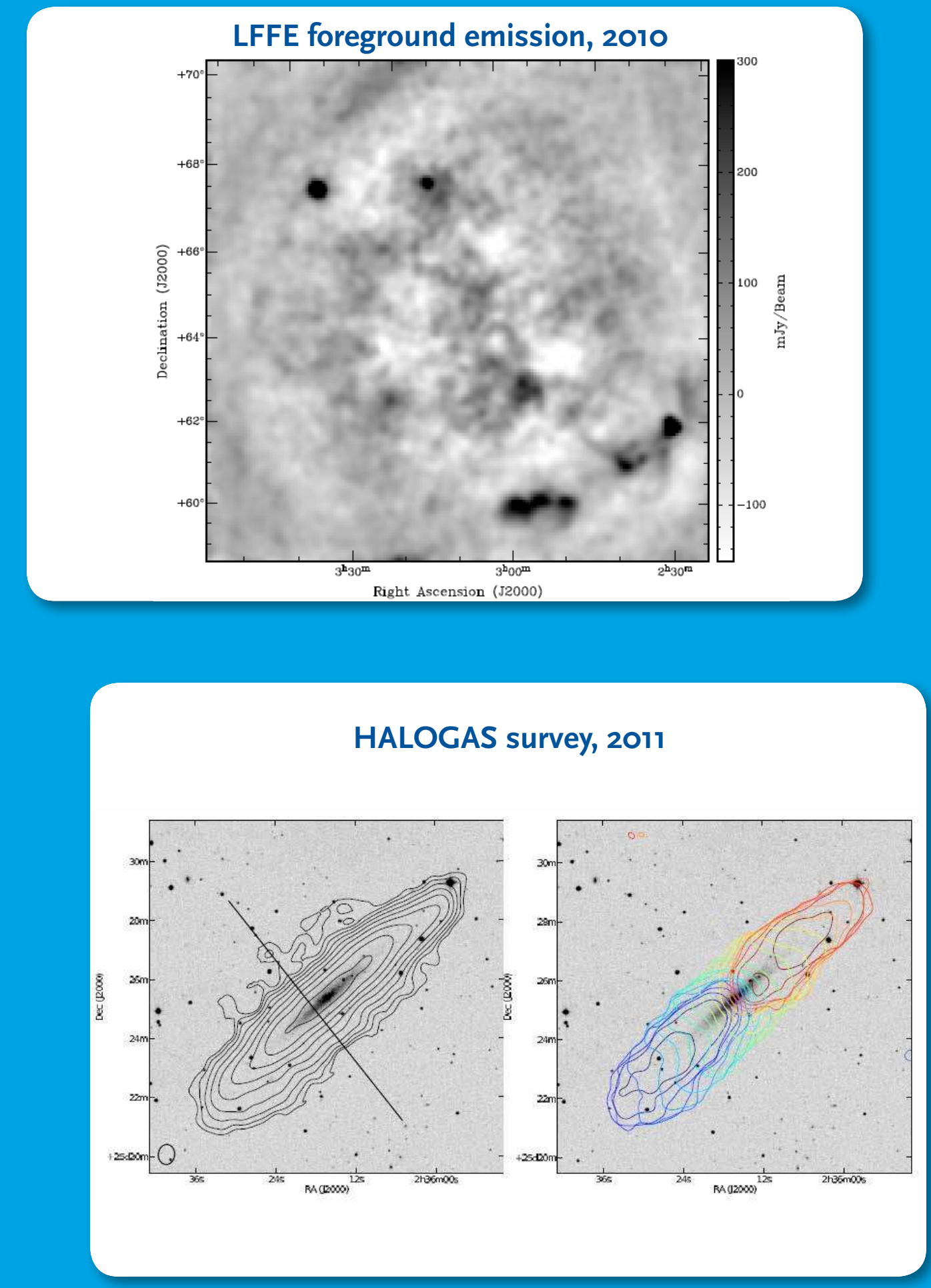

Pulsar in three-body system, 2013

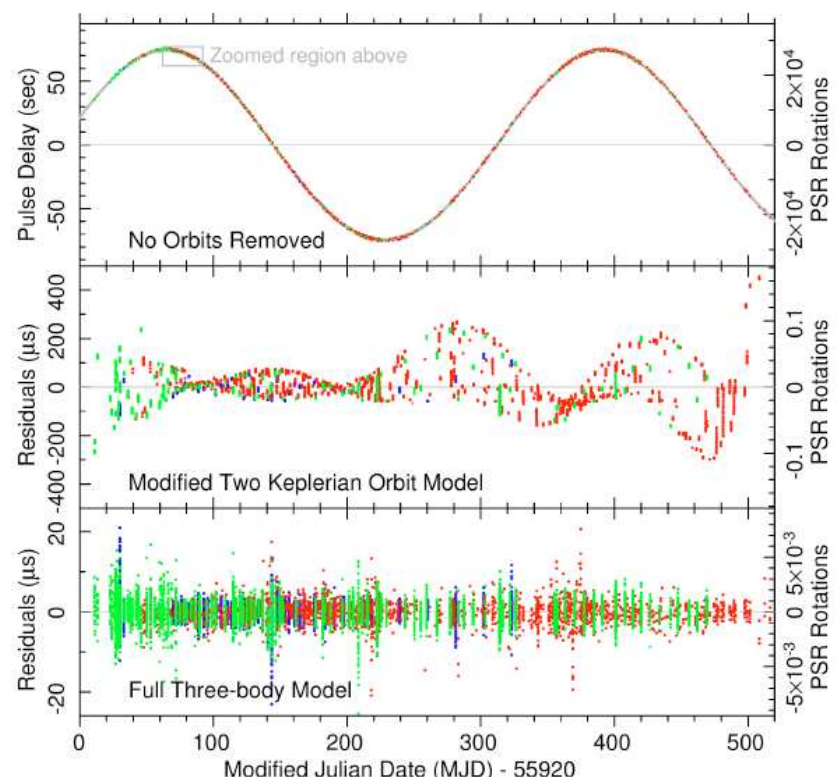

Extraplanar gas in a galaxy, 2013

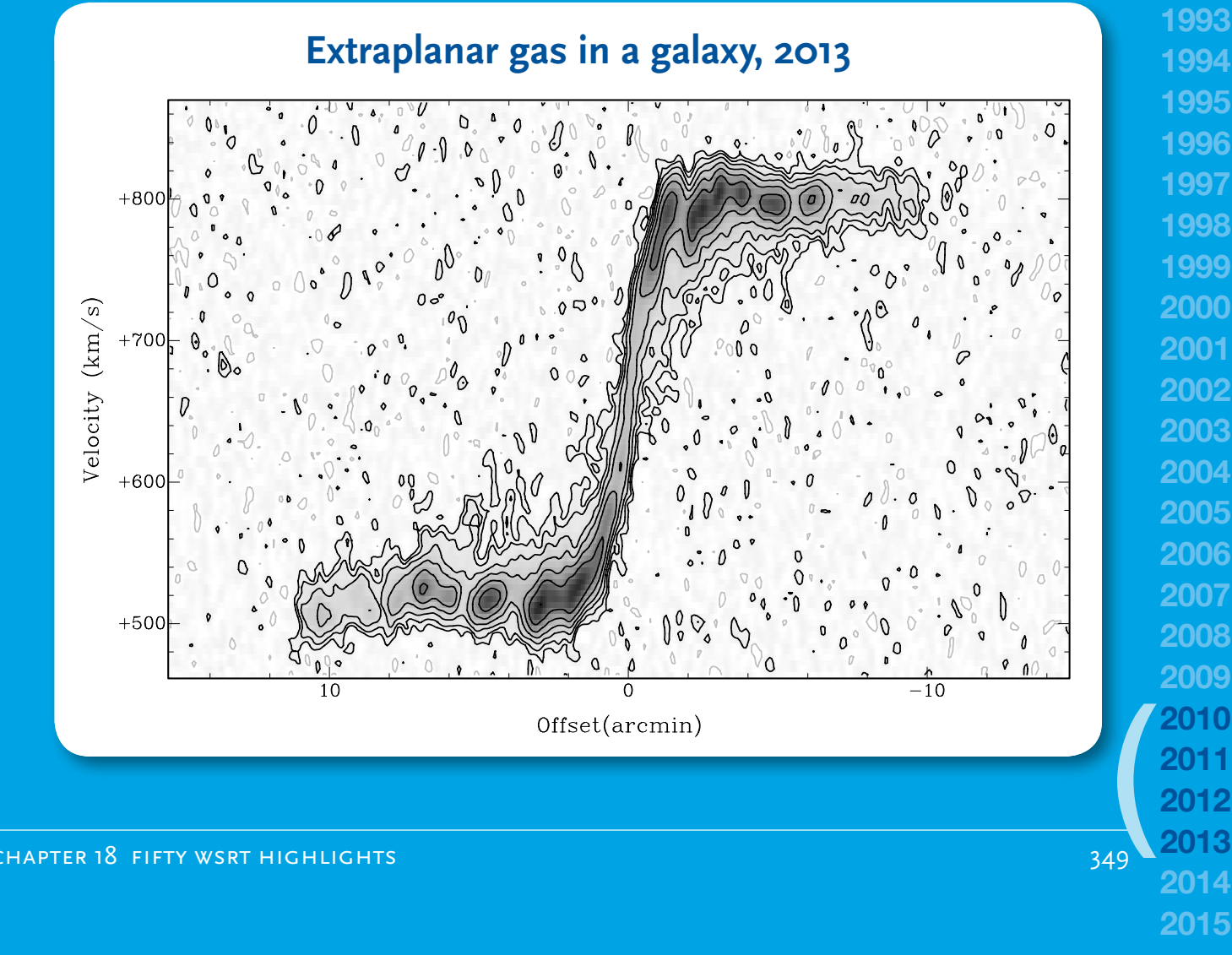




\section{HI stripping in cluster galaxies, 2014}
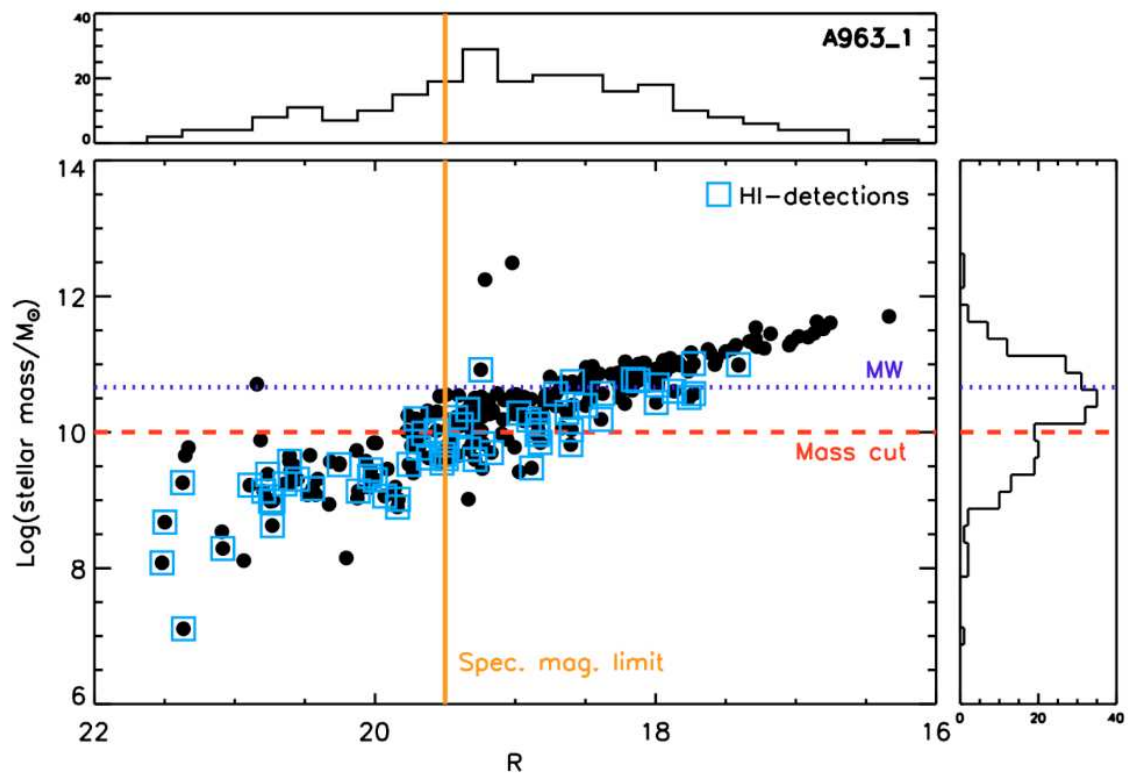

1975

1976

1977

1978

1979

1980

1981

1982

1983

1984

1985

1986

1987

1988

1989

1990

1991

1992

1993

The HI gas absorption zoo, 2015
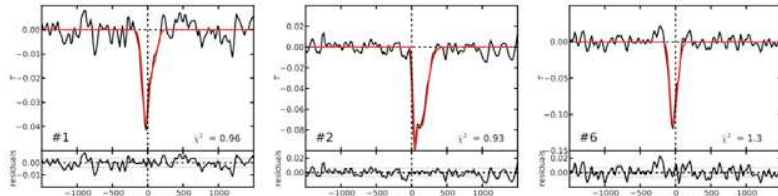

1994

1995

1996

1997

1998
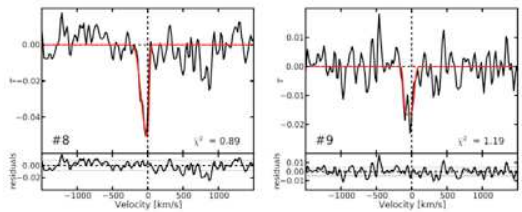

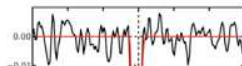

1999

2000

2001

2002
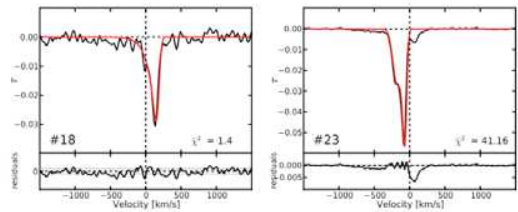

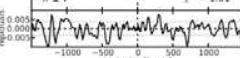

2003

2004

2005

2006

2007

2008

2009

2010 